\title{
Mathematical Model and Experimental Evaluation of Drag Torque in Disengaged Wet Clutches
}

\author{
Shoaib Iqbal, Farid Al-Bender, Bert Pluymers, and Wim Desmet \\ Division PMA, Department of Mechanical Engineering, Katholieke Universiteit Leuven (KU Leuven), Celestijnenlaan 300B, \\ 3001 Heverlee, Belgium
}

Correspondence should be addressed to Shoaib Iqbal; shoaib.iqbal@mech.kuleuven.be

Received 30 May 2013; Accepted 13 July 2013

Academic Editors: J. H. Jang, J. L. Streator, and J. Wang

Copyright (C) 2013 Shoaib Iqbal et al. This is an open access article distributed under the Creative Commons Attribution License, which permits unrestricted use, distribution, and reproduction in any medium, provided the original work is properly cited.

\begin{abstract}
When the clutch is in disengaged condition, ideally no torque should be transmitted. However, in reality, the relative motion between the disks causes viscous shearing of fluids in the gap. This results in a drag torque which is considered as a loss. The objective of the present study is to formulate a drag torque model as well as to experimentally evaluate the effect of several parameters on the drag torque. A model based on continuity and Navier-Stokes equations, considering laminar flow, is deduced. The drag torque estimated by the model is the sum of drag torque due to shearing of the automatic transmission fluid (ATF) and mist (suspension of ATF in air) film. In order to validate the model and characterize the drag torque, experiments are performed using an SAE no. 2 test setup under real conditions of variable ATF flow rate and disks' rotational states for higher clutch speed range. The drag torque predicted by the model is in good agreement with the experimental results obtained by varying the flow properties and disks' rotational states. By analyzing the experimental results, a factor by which, the variation in parameters such as ATF flow rate, ATF temperature, disk size, and disk rotational state influencing the drag torque is determined.
\end{abstract}

\section{Introduction}

When an automatic transmission (AT) is in operation, some of the clutches are engaged to transmit power, while the rest of the clutches remain disengaged. During engagement and disengagement processes, frictional heat is generated which causes wear and deterioration of the disks. Therefore, the disks must be cooled in order to increase the service life of the clutch. For this purpose, ATF is continuously supplied to the disks, irrespective of the state of the clutch. In addition, both the friction disk (FD) and the separator disk (SD) sets are always rotating. The relative motion between the disks causes viscous shearing of fluids film in the gap between the disks. Fluids in the present study collectively refer to the ATF and the mist. This results in a drag torque on both the disks. Depending upon the configuration of the clutches in an AT, the drag torque transmitted by the open clutches is considered as a loss. This unwanted drag torque reduces efficiency and increases temperature of the transmission. The increased temperature promotes the wear of the gears, bearings, and the aging of the ATF. Currently,
AT designers are concerned about how to reduce the drag torque in disengaged wet clutches in order to overcome its undesirable effects. To achieve this reduction, a good understanding of the physical phenomenon and drag torque characteristics of disengaged wet clutches, through modeling and experimentation, is necessary.

In past few decades several models and experimental observations have been reported in order to understand the issue of drag torque in disengaged wet clutches. Some of the renowned drag torque models are briefly discussed as follows. Kitabayashi et al. [1] presented a theoretical model based on laminar flow in the gap between the disks. However, the model does not take into account the rupture of the full ATF film at high speed; hence, it is suitable for predicting only the rising portion of the typical drag torque curve. This drawback was overcome by a model proposed by Kato et al. [2]. They have considered the rupture of the full ATF film due to cavitation at high-speed region in their model. Their formulation shows that the rupture of the ATF film starts from the inner radius. However, subsequent studies suggest that laminar flow shall prevail in the gap between 
the disks, and the rupture of the full ATF film should commence from outer radius and progress towards inner radius. Later, Yuan et al. [3] introduced an additional term in Kato's pressure distribution equation to take into account ATF surface tension effect. In addition, in their formulation, the rupture of the full ATF film starts from the outer radius in inward direction. A concept of equivalent outer radius of the full ATF film in function of speed is introduced by them in order to estimate the drag torque. Moreover, they hypothetically proposed the rivulet shape of the partial ATF film and suggested that the ATF should wet both of the disks' surfaces due to surface tension. Aphale et al. [4] presented a mathematical model based on asymptotic treatment of the Navier-Stokes equations and verified it by computational fluid dynamics (CFD) model and experiments. Initially, they formulated the model by assuming nongrooved disks, but eventually approximated the model for the grooved disks by multiplying the obtained drag torque with the fraction of area which is not grooved in relation to total disk contact area. More recently, Yuan et al. [5] proposed a model based on the Navier-Stokes equations. Through experiments, they showed that for a constant feeding flow rate, the rupture of the ATF film begins from the outer radius and extends towards the inner radius. Mist is formed and the ATF flows only along the rotating disk after rupture. Their model takes into account only the continuous ATF film for the estimation of the drag torque. Yuan et al. [6] developed a two-phase flow CFD model using volume of fluid (VOF) technique for simulating the drag torque in wet clutches. They predicted the ATF cavitation mode, flow pattern, and drag torque through simulations for different disks' rotation conditions.

Although quite a large amount of work related to drag torque model development has been reported so far, the research concerning the drag torque experimental findings is still lacking. Some of the experimental observations were first reported by Schade [7]. Later, Fish [8], Lloyd [9], and Kitabayashi et al. [1] contributed to the topic. They studied the effect of parameters such as ATF flow rate, ATF viscosity, gap height, disks' waviness, friction material, and groove pattern on the drag torque.

A review of the previous studies, collectively in modeling and experimental domains, suggests that a constant ATF flow rate is considered. However, in real operation, the ATF flow rate increases with increasing speed. Consequently, there exists a significant amount of ATF film in the ruptured section after cavitation, which is unfortunately ignored by most of the models. As a result, the combined contribution of the ATF and mist film towards the development of drag torque is unaccounted. In addition, in these studies only one set of disks is rotating while the other set is stationary, but in reality both sets of disks can rotate with different speeds and directions simultaneously. Furthermore, in most of the studies, the lower speed range of $2000-2500 \mathrm{rpm}$ is considered, whereas in reality, the speed range can go higher up to $4500-5000 \mathrm{rpm}$, when the clutch is associated with top gears. Moreover, in many studies, a single pair of disks is considered instead of multidisks configuration.

The aim of this study is twofold. Firstly, to develop a drag torque mathematical model and validate the model using the experimental results; secondly, to experimentally characterize the drag torque in disengaged wet clutches, under real conditions of variable flow rate and disk rotational state for higher clutch speed range.

The paper is organized as follows. Section 2 describes the formulation of the model. In this section, flow velocity and pressure field are deduced from basic flow equations. In order to account for variable flow rate, the model considers the ATF (both in the continuous and ruptured sections) and the mist film in the gap between the disks.The model quantifies the volume fraction of the fluids and finally estimates the drag torque. The experimental aspects comprising the description of the advanced SAE no. 2 test setup, and the drag torque test procedures are presented and discussed in Section 3. Section 4 is dedicated to the model validation. The correlation between the experimental and the model simulation results are presented and discussed in this section. Section 5 focuses on the experimental characterization of the drag torque. The influence of variation in parameters such as ATF flow rate, ATF temperature, disks' size, and disks' rotational states on the drag torque is studied. By examining the experimental results, the relationships between the amount by which the parameters are varied and the corresponding change in drag torque are established. Finally, some important conclusions drawn from the study are summarized in Section 6.

\section{Model Development}

The flow velocity field and the associated pressure distribution field in the gap between the disks are obtained by simplifying the continuity and Navier-Stokes momentum equations. The achieved flow velocity and pressure fields are then utilized to solve the flow development. This leads to the development of the model which is described in this section.

Figure 1 depicts the basic geometry of the single pair of clutch disks along with flow configuration. For the ease of illustration, only half of the disks are shown. It shows two annular-shaped smooth disks separated by a uniform gap $h$, having inner and outer radii $r_{i}$ and $r_{o}$, respectively. In addition, the upper and lower disks are located at an axial distance of $z=0$ and $z=h$. These upper and lower disks resemble the FD and SD, respectively. Furthermore, the upper and lower disks are rotating about the shaft $(z$ axis) with an angular speed of $\omega_{1}$ and $\omega_{2}$, respectively. The radial, circumferential, and axial coordinates are $r, \theta$, and $z$, respectively. The flow direction is indicated by the arrows. The ATF is fed into the gap between the disks through a hollow passage in the hub, by means of a pump. The ATF enters the gap at the inner radius and thereafter flows radially through the gap between the disks and discharges at the outer radius, where ambient pressure $P_{a}$ prevails. $Q_{a}$ is the actual flow rate in the gap between the disks. The fluid pressure at the inner radius is termed supply pressure $P_{s}$ and is determined by the pump flow rate $Q_{a}$ and the impedance to the flow in the gap. (In other words, we assume zero impedance to the flow upstream of the inlet radius.) The ATF film is not always full and continuous but gets ruptured with the formation of 

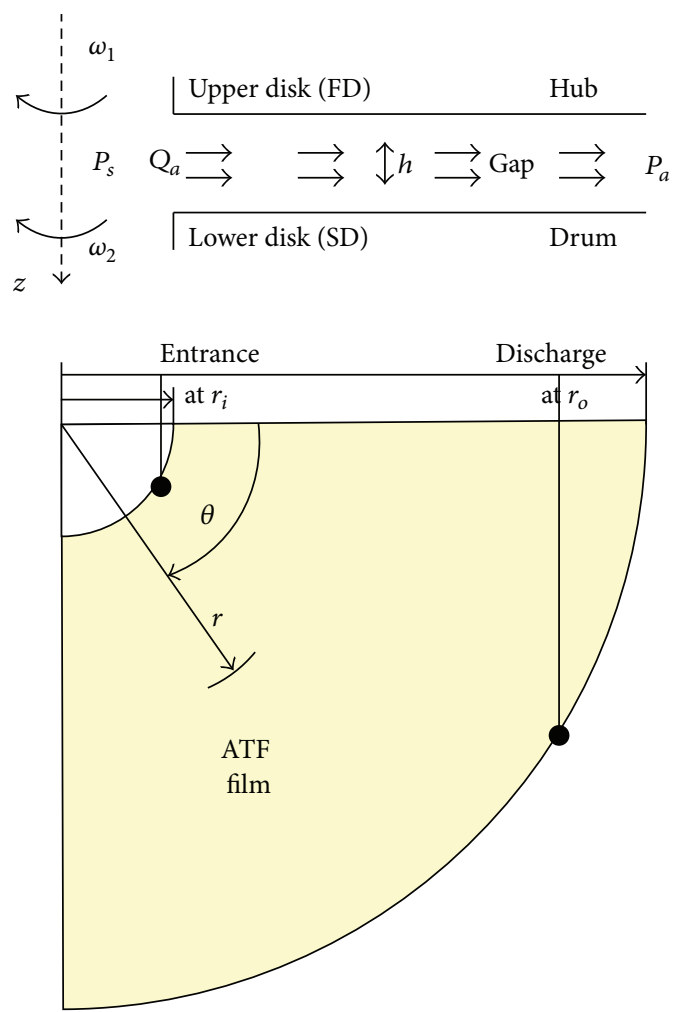

FIGURE 1: Schematic of the clutch geometry (single pair of disks) along with flow configuration.

rivulet due to cavitation at higher clutch speeds. In general, all the flow parameters are known (with certain assumptions), except the radial extent of the continuous ATF film $\left(r^{*}\right)$ at high clutch speed region. Thus, the problem is to determine $r^{*}$, which is essential for estimating the drag torque.

2.1. Reduced Continuity and Navier-Stokes Equations. Several levels of simplification of the basic continuity and NavierStokes equations in the gap between the disks of the wet clutches are possible. They fall into three main categories: assumptions based on the type and nature of flow; approximation based on idealizations of the fluid properties and geometry; truncation of the describing equations based on the order of magnitude evaluation of the various terms. After applying the first and second simplifications, the describing equations are referred to as the reduced flow equations. The first two simplifications are described below, whereas the third simplification will follow later.

The flow is viscous, apparent from the fact that the resistance offered by the gap to the fluid gives rise to shear force. In addition, the flow is laminar on the basis of the Reynolds number (less than 2000) typically observed in open wet clutches $[3,5]$. Furthermore, the flow is treated as steady when assuming that the flow geometry, feed rate, and boundary conditions do not change in time. Moreover, the flow is considered axis-symmetrical about the $z$-axis owing to the specific configuration of flow. The body forces generated by the action of gravitation are assumed negligible compared to the other forcing terms in the flow equation. In general, the physical properties of a fluid are dependent on temperature and to a lesser degree on pressure. However, considering small temperature and pressure variation of the fluid in the gap between the disks, the fluid's physical properties such as density $(\rho)$ and dynamic viscosity $(\mu)$ may be taken to be constant. Thus, the fluid is treated as incompressible. Typically in wet clutches $h / r_{o} \ll 1$ (where, e.g., gap height $h=1 \mathrm{~mm}$ and outer radius $r_{o}=100 \mathrm{~mm}$ ). This suggests that the fluid film in the gap between the disks is thin in the axial $z$ direction. Hence, the flow velocity in the axial direction may be neglected.

Based on aforementioned simplifications, the reduced continuity and momentum equations written in cylindrical coordinates $(r, \theta, z)$ (relevant to our configuration), and denoting the corresponding velocity vectors by $\left(V_{r}, V_{\theta}, V_{z}\right)$, are as follows [10]:

$$
\begin{gathered}
\frac{\partial V_{r}}{\partial r}+\frac{V_{r}}{r}=0 \\
V_{r} \frac{\partial V_{r}}{\partial r}-\frac{V_{\theta}^{2}}{r}=-\frac{1}{\rho} \frac{\partial p}{\partial r}+\frac{\mu}{\rho}\left(\frac{\partial^{2} V_{r}}{\partial r^{2}}+\frac{1}{r} \frac{\partial V_{r}}{\partial r} \frac{\partial^{2} V_{r}}{\partial z^{2}}-\frac{V_{r}}{r^{2}}\right) \\
V_{r} \frac{\partial V_{\theta}}{\partial r}+\frac{V_{r} V_{\theta}}{r}=\frac{\mu}{\rho}\left(\frac{\partial^{2} V_{\theta}}{\partial r^{2}}+\frac{1}{r} \frac{\partial V_{\theta}}{\partial r}+\frac{\partial^{2} V_{\theta}}{\partial z^{2}}-\frac{V_{\theta}}{r^{2}}\right) \\
0=-\frac{\partial p}{\partial z}
\end{gathered}
$$

where the inertial terms are set on the left-hand side. The last equation of the set (4) means the pressure is constant across the ATF film.

Within the disks, the no-slip boundary conditions apply to the flow-velocity components at the surfaces as follows:

$$
V_{r}(r, \theta, 0)=0, \quad V_{r}(r, \theta, h)=0 .
$$

Moreover, with the disks rotating at angular speeds of $\omega_{1}$ and $\omega_{2}$, respectively, we have

$$
V_{\theta}(r, \theta, 0)=r \omega_{1}, \quad V_{\theta}(r, \theta, h)=r \omega_{2} .
$$

Auxiliary conditions are

$$
P\left(r_{o}, \theta, z\right)=P_{a}=0 ;
$$

and the flow rate through the gap is given to be $Q_{a}$.

2.2. Truncation of the Flow Equations. In order to further simplify (2) and (3), truncation is considered on the basis of order-of-magnitude analysis. This is best achieved by normalizing the equations in such a way that all the normalized variables have equal orders of magnitude as reported in [10]. The truncated equations without appreciable loss of accuracy are

$$
\begin{gathered}
-\frac{V_{\theta}^{2}}{r}=-\frac{1}{\rho} \frac{\partial p}{\partial r}+\frac{\mu}{\rho} \frac{\partial^{2} V_{r}}{\partial z^{2}}, \\
0=\frac{\mu}{\rho} \frac{\partial^{2} V_{\theta}}{\partial z^{2}} .
\end{gathered}
$$


2.3. Solution of the Flow Equations. In order to achieve the flow velocity and pressure fields, the flow equations are solved as follows. Integrating (9) with respect to $z$ twice and applying the boundary conditions (6), the flow circumferential velocity distribution in the gap is given by

$$
V_{\theta}=r\left(\Delta \omega \frac{z}{h}+\omega_{2}\right)
$$

where $\Delta \omega=\omega_{1}-\omega_{2}$ is referred to as the clutch speed in present study.

Substituting $V_{\theta}$ from (10) into (8) and rearranging gives

$$
\frac{\partial^{2} V_{r}}{\partial z^{2}}=\frac{1}{\mu} \frac{\partial p}{\partial r}-\frac{\rho r}{\mu}\left(\Delta \omega \frac{z}{h}+\omega_{2}\right)^{2} .
$$

Integrating (11) with respect to $z$ twice and applying the boundary conditions (6), we have the flow radial velocity distribution in the gap as

$$
\begin{aligned}
V_{r}= & \frac{1}{2 \mu} \frac{d p}{d r} z(z-h)-\frac{\rho r}{12 \mu h^{2}} \\
& \times\left(\Delta \omega^{2} z\left(z^{3}-h^{3}\right)+4 \Delta \omega \omega_{2} z h\left(z^{2}-h^{2}\right)\right. \\
& \left.+6 \omega_{2}^{2} z h^{2}(z-h)\right) .
\end{aligned}
$$

The first term on the right-hand side of (12) represents the pressure-induced flow, or Poiseuille flow, while the second term represents the contribution due to the centrifugal force and referred to inertial flow.

The flow rate through the gap between the disks can be obtained from

$$
Q_{a}=\int_{0}^{2 \pi} \int_{0}^{h} V_{r} r d z d \theta=2 \pi r \int_{0}^{h} V_{r} d z
$$

Substituting $V_{r}$ from (12) into (13) and evaluating the quadrature, the actual flow rate can be written as

$$
\begin{aligned}
Q_{a} & =-\frac{\pi r h^{3}}{6 \mu} \frac{d p}{d r}+\frac{\rho \pi r^{2} h^{3}}{6 \mu}\left(\omega_{2}^{2}+\omega_{2} \Delta \omega+\frac{3}{10} \Delta \omega^{2}\right) \\
& \equiv Q_{p}(r)+Q_{c}(r) .
\end{aligned}
$$

The first and second terms on the right-hand side of (14) are the flow rate contribution due to the pressure or Poiseuille force $\left(Q_{p}\right)$ and the centrifugal force $\left(Q_{c}\right)$, respectively. The actual flow rate $Q_{a}$ is given, but the radial extent of the continuous ATF film $\left(r^{*}\right)$ in function of clutch speed is unknown. The way by which $r^{*}$ is estimated is described in the next section.

Rearranging (14), an expression for the pressure gradient of the flow in the gap between the disks is obtained as

$$
\frac{d p}{d r}=-\frac{6 Q_{a} \mu}{\pi r h^{3}}+\rho r\left(\omega_{2}^{2}+\omega_{2} \Delta \omega+\frac{3}{10} \Delta \omega^{2}\right) .
$$

Finally, integrating (15) with respect to $r$ and applying the auxiliary condition (7) yields the flow pressure distribution in the gap between the disks, given by

$$
p(r)=-\frac{6 Q_{a} \mu}{\pi h^{3}} \ln \frac{r_{o}}{r}+\frac{\rho\left(r_{o}^{2}-r^{2}\right)}{20}\left(3 \omega_{1}^{2}+3 \omega_{2}^{2}+4 \omega_{1} \omega_{2}\right) \text {. }
$$

2.4. Flow Development. In general, at any instant, the flow configuration depends upon the magnitude of the Poiseuille and centrifugal forces. However, in low clutch speed region, the flow is largely governed by the Poiseuille force. In such a situation, there exists a full continuous ATF film in the gap between the disks. With the increase in clutch speed, the centrifugal force increases. After reaching certain speed, the centrifugal force dominates the Poiseuille force in determining the flow. According to (14), with increasing clutch speed, the flow rate contribution due to the centrifugal force $\left(Q_{c}\right)$ increases. As the actual flow rate $\left(Q_{a}\right)$ remains constant (see (14)), this implies that in order to satisfy continuity the flow rate contribution due to the Poiseuille force $\left(Q_{p}\right)$ must decrease with a limiting value of zero resulting in the cavitation. The increased centrifugal force acting on the ATF film causes cavitation $[2,6]$. Due to the cavitation, the ATF film is no longer continuous but gets ruptured starting from the outer radius in inward direction with the formation of rivulets [3] (see Figure 2(a)). In between the rivulets there exists a mist film [5]. In order to estimate the radial extent of the continuous ATF film, we must apply boundary conditions at the inception of the cavitated region (interface between the continuous ATF and mist film).

The continuity analysis leads to the condition that the pressure gradient at the inception of the cavitated region must be zero. This pressure condition is known as the Reynolds cavitation boundary condition [11] and is mathematically expressed by

$$
\frac{d p}{d r}=0, \quad p_{\text {cav }}=p_{a}=0
$$

Because of the fact that the clutch is open to the atmosphere, the pressure in the cavitating region $\left(p_{\text {cav }}\right)$ is equal to the ambient pressure (assumed to be zero in the present study).

The radial extent of the continuous ATF film or the radial location of the inception of the cavitation $\left(r^{*}\right.$ as shown in Figures 2(b) or 2(c)) is found by applying the Reynolds cavitation boundary condition to (14) which results in

$$
Q_{a}=\frac{\rho \pi r^{2} h^{3}}{6 \mu}\left(\omega_{2}^{2}+\omega_{2} \Delta \omega+\frac{3}{10} \Delta \omega^{2}\right)
$$

From (18), $r^{*}$ is found as a function of clutch speed.

There are two possibilities of the behavior of the ruptured section of the ATF film. Firstly, it could be assumed to make contact with both of the disks with the formation of rivulets [3]. Secondly, it could be assumed to detach itself from one of the two disk surfaces, making contact with only one disk [5]. In the present study, we will consider that the ATF film in the ruptured section maintains contact with both the disk surfaces.

Now, consider a sector of the cavitating fluid film with an angle $\alpha$ as shown in Figure 2(c). The fraction of the ATF film in Figures 2(a) and 2(b) is the same. For the ease of the quantification of the ATF and mist film, in the later figure, the flow is aligned in the radial flow direction instead of the actual flow direction. The "wetted" fraction $(\phi(r))$ of the ATF film in 


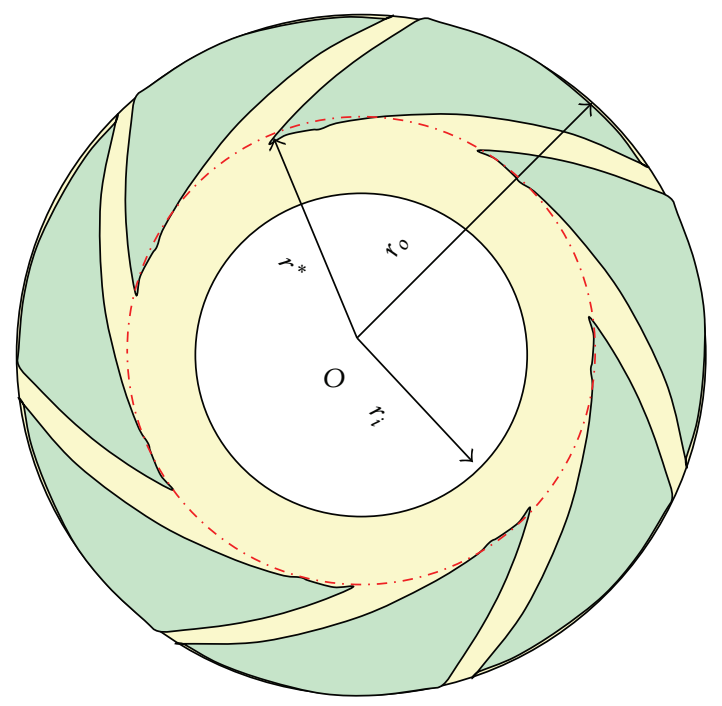

(a)

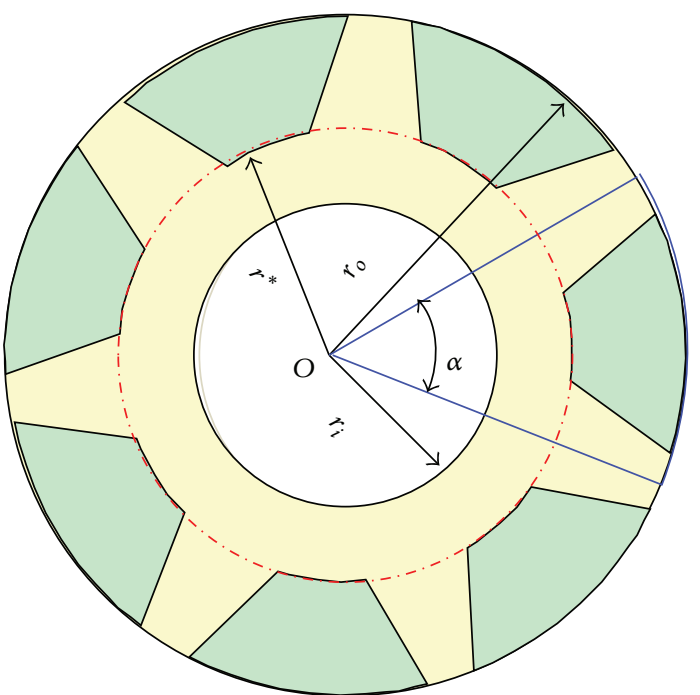

(b)

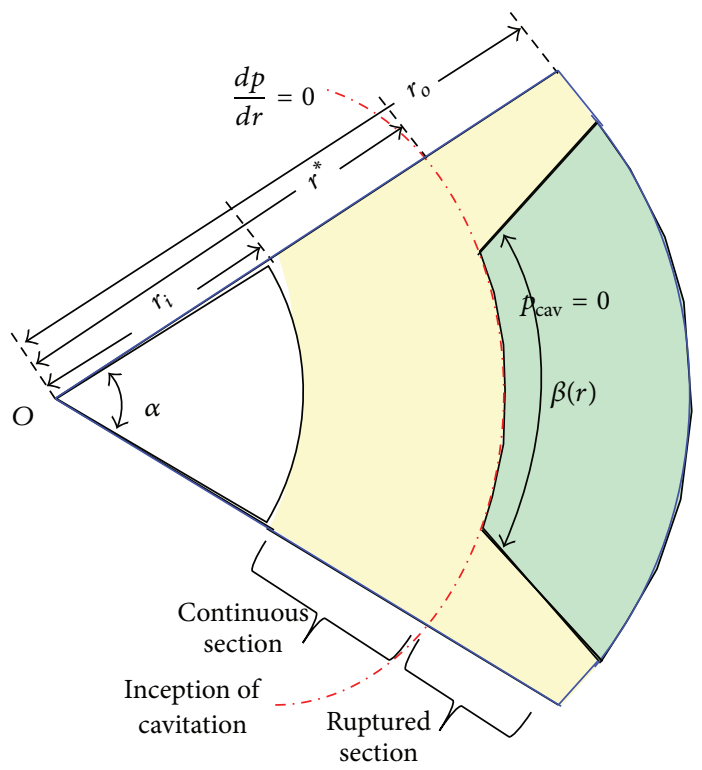

(c)

FIGURE 2: Schematic of the film shape: (a) complete film (actual direction), (b) complete film (radial direction), and (c) a sector of the complete film (radial direction); yellow and green portions represent the ATF and the mist film, respectively.

the ruptured section with respect to the full continuous ATF film can be written as

$$
\phi(r)=\frac{\alpha-\beta(r)}{\alpha},
$$

where $\alpha$ is an arbitrarily chosen sector angle and angle $\beta(r)$ represents the mist film angle considering the radial flow.

Next, from equality of the flow in the continuous and the ruptured section $\left(Q_{p}=0\right)$, we get

$$
\begin{gathered}
Q_{a}=\phi(r) \cdot 2 \pi r \int_{o}^{h} v_{r} d z=\phi(r) \cdot Q_{c}, \\
\phi(r)=\frac{Q_{a}}{Q_{c}}=\frac{6 \mu Q_{a}}{\rho \pi r^{2} h^{3}}\left(\frac{1}{\omega_{2}^{2}+\omega_{2} \Delta \omega+(3 / 10) \Delta \omega^{2}}\right) .
\end{gathered}
$$

$\phi(r)$ can be estimated by varying $r$ from $r^{*}$ to $r_{o}$ in (21) as a function of clutch speed. By substituting the obtained $\phi(r)$ from (21) in (19), angle $\beta(r)$ can be evaluated.

In case of constant actual flow rate $Q_{a}$, the centrifugalflow component $Q_{c}$ increases with increasing clutch speed. Thus, the fraction $\phi(r)$ must reduce in order to satisfy the flow equality. This means that the ATF film will become thinner $(\beta(r)$ will increase) with increasing clutch speed but never vanishes. In addition, at a particular clutch speed, the ATF film in the ruptured section will get thinner towards the outer radius, which is apparent from $(21)(\phi(r)$ proportional to $\left.1 / r^{2}\right)$. In the ruptured section, if $\phi(r)$ is the fraction of the ATF film, then remaining $(1-\phi(r))$ is the fraction of the mist film. 
2.5. Drag Torque Estimation. The drag torque is estimated by considering that the fluids film is in contact with both of the disks. Therefore, the total drag torque is the sum of the drag torque contribution due to ATF film in the continuous section and the ATF and the mist film in the ruptured section as written below [10].

The contribution due to the ATF film in continuous section is

$$
T_{f a}=\int_{0}^{2 \pi} \int_{r_{i}}^{r^{*}} N r \tau_{z \theta} r d r d \theta=\frac{\pi \mu_{\mathrm{ATF}} \Delta \omega N}{2 h}\left(r^{* 4}-r_{i}^{4}\right) .
$$

is

The contribution due to the ATF film in ruptured section

$$
\begin{aligned}
T_{r a} & =\int_{0}^{2 \pi} \int_{r^{*}}^{r_{o}} N \phi(r) r \tau_{z \theta} r d r d \theta \\
& =\frac{2 \pi \mu_{\mathrm{ATF}} \Delta \omega N}{h} \int_{r^{*}}^{r_{o}} \phi(r) r^{3} d r .
\end{aligned}
$$
is

The contribution due to the mist film in ruptured section

$$
\begin{aligned}
T_{r m} & =\int_{0}^{2 \pi} \int_{r^{*}}^{r_{o}} N(1-\phi(r)) r \tau_{z \theta} r d r d \theta \\
& =\frac{2 \pi \mu_{\mathrm{mist}} \Delta \omega N}{h} \int_{r^{*}}^{r_{o}}(1-\phi(r)) r^{3} d r .
\end{aligned}
$$

The model is approximated for the grooved FDs. Since the grooves tend to reduce the contact area, a good engineering approximation to the problem will be to reduce the drag torque by area ratio $(\gamma)[4]$. Area ratio is defined as the ratio of ungrooved area to the total disk area. Finally, the total drag torque is given by

$$
T=\gamma \cdot\left(T_{f a}+T_{r a}+T_{r m}\right)
$$

\section{Experimental Aspects}

This section begins with description of the clutch package and the SAE no. 2 test setup used in the study. The procedures for carrying out drag torque experiments are also discussed. Finally, the test specifications are given in the last part of this section.

3.1. Configuration of the Clutch Package. Cross-sectional and exploded views of the clutch package are shown in Figure 3. It consists of a hub (1) and a drum (2). SDs (3) are mounted to the drum by splines, and the FDs (4) are mounted to the hub by lugs. The SDs and the FDs core are typically made from plain carbon steel, whereas the FDs are lined on both sides by paper-, bronze-, or carbon-fiber-based materials. The engagement process begins when the clutch is actuated by supplying the ATF under pressure which produces the normal load acting on the piston (5) against the spring (6) for engaging the disks. The actuating ATF (shown by red line in the figure) flows through the internal hole (7) directed towards the piston. Once the clutch is fully locked or relative velocity between the driver and driven members is zero, the power is transmitted through shafts via the drum and the hub. ATF is also supplied continuously, irrespective of the state of the clutch (closed or open). The purpose of this continuous supply of ATF is to cool the disks, maintain the disks' surfaces clean, and provide smoother performance and longer life time. The cooling ATF enters the clutch through the internal holes (8) in the shaft and then flows into the gap between the disks (shown by the green line in the figure). Afterwards, it flows outward in the radial direction by pressure and centrifugal forces and then flows back into the reservoir through the holes in the drum. In the present study, we are concerned with the flow of the cooling ATF, since we are dealing with the wet clutches in open condition.

3.2. An Advanced SAE No. 2 Test Setup. The drag torque tests are performed on an advanced SAE no. 2 test setup designed and built by industrial partner, Dana Spicer Off Highway, Belgium as shown in Figure 4. The SAE no. 2 test setup is called "advanced" in the sense that both sets of SDs and FDs can rotate independently. In case of a previous version of the SAE no. 2 test setup, only one set of disks were able to rotate while the other set were held stationary. The test setup basically consists of three main systems: driveline, control, and measurement system. The driveline consists of nine components: input and output $30 \mathrm{~kW} \mathrm{AC}$ motor for independently driving the input and the output members through pulley-timing belt transmission (1 and 8), input and output speed sensors, based on hall effect encoder comprising sensing gear having 51 teeth (2 and 7), input flywheel (drum side containing SDs) (3), output flywheel (hub side containing FDs) (6), multidisk clutch (4), and torque sensor (5) based on optical-electronic light method which senses the twisting of the torsion shaft. It comprises two slotted disks. The light is passed through the disks; as the disks rotate relatively to one another, the transmission ratio of the light changes proportionally to the torque.

The hydraulic circuit of the test setup is discussed as follows. A heat exchanger installed in the main tank allows the tests to be performed at the elevated ATF temperature as experienced in real operation. Thermocouples measure the ATF temperatures at the inlet and outlet of the clutch near the housing. The centrifugal pump feeds the ATF from the main tank at a flow rate (set as per test condition) to the main line. The flow pressure rises due to pumping action. A pressure relief valve installed in the main line helps in maintaining the flow pressure to the required level. The excess ATF is diverted to the main tank through an auxiliary line. The flow rate circulated to the clutch package is further adjusted by the restrictor valve according to the signal obtained by the flow meter. A pressure transducer (based on deformation of a membrane) records the flow pressure near to the housing. The outlet flow from the clutch is temporarily stored in the outlet tank which is pumped to the main tank after filtration for recirculation. An integrated control system is used for controlling the ATF flow rate, flow temperature, and the desired rotational speed of the disks. 


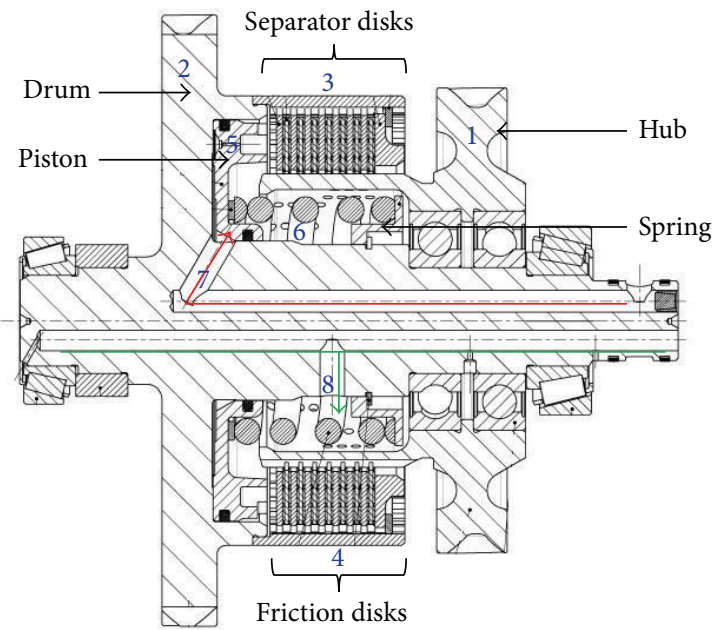

(a)

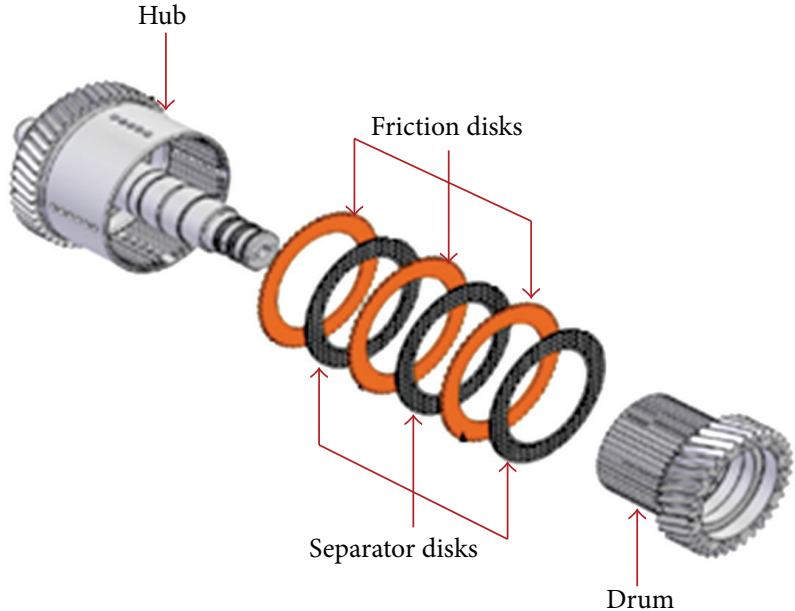

(b)

FIGURE 3: Schematic of the clutch package: (a) cross-sectional view; (b) exploded view.

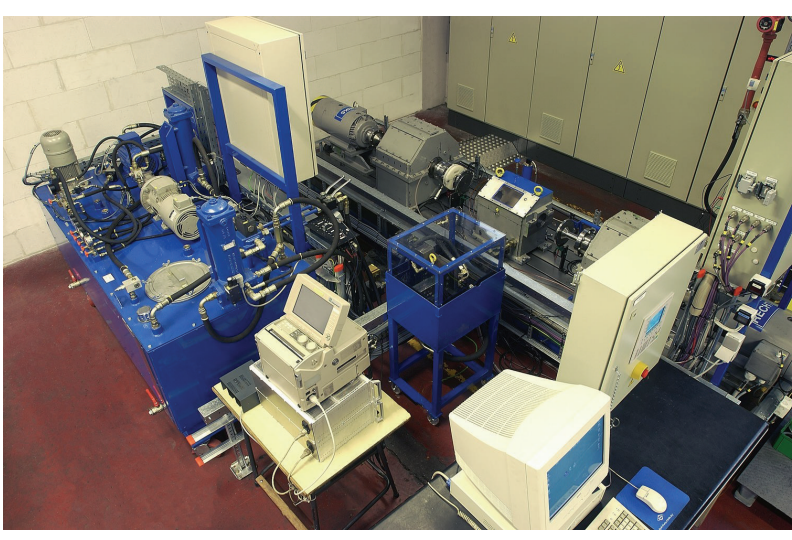

(a)

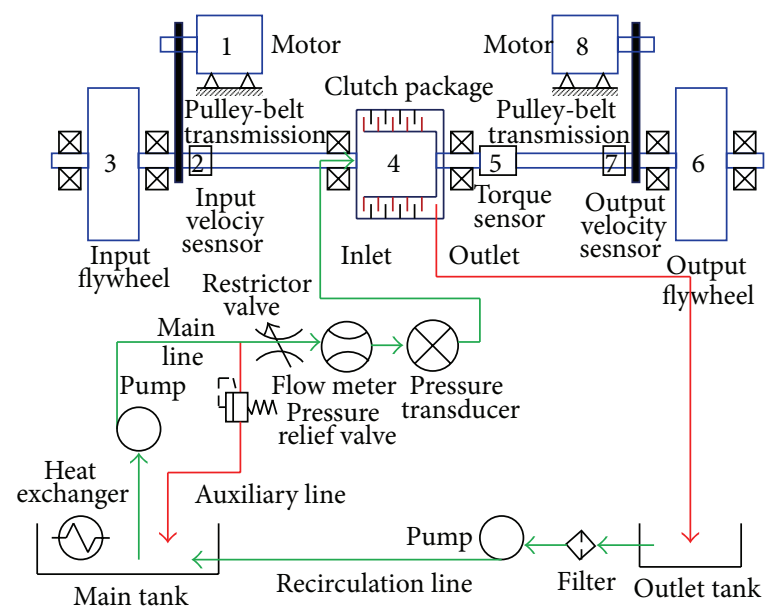

(b)

FIGURE 4: An advanced SAE no. 2 test setup: (a) actual photograph (Courtesy Dana SOHB); (b) schematic diagram along with hydraulic circuit.

3.3. Drag Torque Experimental Procedure. A new clutch package undergoes a run-in test for 100 cycles, before the drag torque test is carried out. This is done in order to achieve the stabilized friction characteristics of the clutch. The run-in test procedure is in principle the same as that of engagement cycle test [12], but the starting relative velocity (input energy) for the run-in test is kept lower than that of the engagement test. This is done to avoid any thermal failure of the clutch due to the development of excessive braking energy.

The drag torque test is performed with run-in disks as follows. FDs are mounted to the hub by lugs, whereas the SDs are mounted to the drum by splines with a desired gap height between the disks bounded by the end stopper, enclosed in a metal housing. The inertia of each flywheel is set to $0.5018 \mathrm{kgm}^{2}$. This inertia provides an energy level of a typical light throttle engagement for a shifting clutch. Since the drag torque test is performed in an open condition of the clutch, the ATF supply for actuation or clutch engagement is shut off. After the adjustment of the required flow conditions (cooling ATF flow rate and temperature), the disks are rotated to the required speed and direction. After reaching the required level, the disks are rotated for another $5 \mathrm{~s}$ in order to stabilize the drag torque before initiating the measurement. After stabilization time period, the data are recorded for $5 \mathrm{~s}$ and the average value is considered. The repeatability of the experiments is tested for three runs. The physical parameters measured during the tests, and their relevant sensors are listed in Table 1. The data are captured with the sampling frequency of $10 \mathrm{~Hz}$.

3.4. Test Specifications. Experiments are performed in order to validate the model as well as to evaluate the influence 
TABLE 1: Test parameters.

\begin{tabular}{llc}
\hline Physical parameters & Sensor type & Unit \\
\hline Torque & Optical electronic light sensor & $\mathrm{Nm}$ \\
Velocity hub (FD) & Optical encoder & $\mathrm{rpm}$ \\
Velocity drum (SD) & Optical encoder & $\mathrm{rpm}$ \\
ATF flow rate & Flow meter & $\mathrm{L} \cdot \mathrm{min}^{-1}$ \\
ATF pressure & Pressure transducer & $\mathrm{Bar}$ \\
ATF temperature & Thermocouple & ${ }^{\circ} \mathrm{C}$ \\
\hline
\end{tabular}

of several parameters on the drag torque. The parameters considered in this study are ATF flow rate, ATF temperature, disk size, and disk rotational state. The test specifications are derived keeping in mind the nominal value of designed parameters encountered in actual condition of an AT in tractors and earth moving vehicles. Except for the case of disk rotational state, for all the tests, FDs are rotated while the SDs are held stationary (FS0). In case of disk rotational state, the influence of rotational state of the disks (rotational speed and direction) on the drag torque is assessed. For this purpose, three tests are conducted, namely, FS0, ER (SDs and FDs are rotated with same constant speed and in same direction), and OR (SD's and FD's are rotated with same constant speed, but in opposite direction).

For all the tests, the real conditions of variable flow rate and higher clutch speed range are imposed. Moreover, the lining material of the FDs is paper based having waffle pattern, while the material of the FDs core and the SDs is plain carbon steel.

Variable flow rate means that the flow is varied in a manner depicted in Figure 5. This means that up to a clutch speed of $\Delta \omega_{f}$, the flow rate has fixed initial value $Q_{i}$. Beyond $\Delta \omega_{f}$, the flow rate increases with the rate governed by the slope $(m)$ according to the formula shown by

$$
Q_{a}= \begin{cases}Q_{i}, & \Delta \omega \leq \Delta \omega_{f}, \\ Q_{i}+m\left(\Delta \omega-\Delta \omega_{f}\right), & \Delta \omega>\Delta \omega_{f} .\end{cases}
$$

When one set of disks is rotating while the other set is stationary (FS0), $\Delta \omega_{f}$ is $1000 \mathrm{rpm}$. In contrast, when both the sets of disks are rotating (ER, OR), $\Delta \omega_{f}$ changes to $2000 \mathrm{rpm}$.

The clutch geometrical details and the test specifications for model validation and experimental evaluation of the drag torque are summarized in Tables 6 and 7, respectively.

\section{Model Validation}

To better demonstrate the model capabilities, the simulations results are compared with the experimental results. Experiments are designed under real conditions of variable flow rate and disk rotational state, considering higher clutch speed range. For all the tests cases, the dynamic viscosity of the mist is unknown. Consequently, this has to be postulated on a reasonable basis knowing the fact that at high speed region, the drag torque is governed by the mist film. In addition, not the entire supply flow is expected to pass into the gap between the disks: some amount of fluid which flows around the clutch housing and outside the radial extent of the disks constitutes

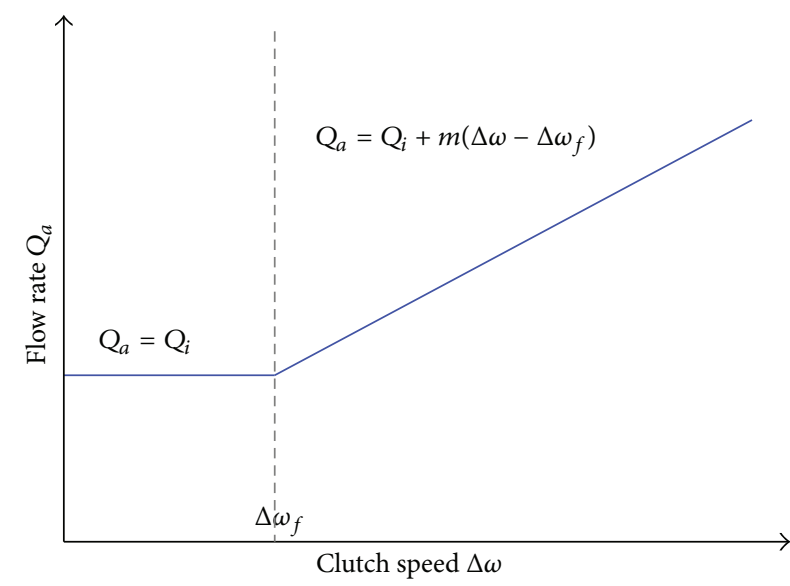

FIGURE 5: Variation of the flow rate with clutch speed.

a so-called ineffective flow. Therefore, the actual flow rate in the gap between the disks is estimated from the supply flow rate, keeping in mind the ineffective flow and any additional leakage in the flow circuit. To serve the purpose, a reduction factor is determined using the clutch package geometrical details. The reduction factor is defined as a ratio of effective volume to the total volume of the clutch package.

4.1. ATF Flow Rate. In order to assess the model behavior for different magnitude of flow rate, two tests are performed. For these two tests, the initial values of flow rate are 2.2 and $4 \mathrm{~L} \cdot \mathrm{min}^{-1}$, respectively. The remaining common parameters are given in Table 7 , test case 1 , belonging to the model validation group. The ATF density is $885 \mathrm{Kg} \cdot \mathrm{m}^{-3}$. The ATF and mist dynamic viscosities considered for the simulations are 0.070 and $0.0045 \mathrm{~Pa} \cdot \mathrm{s}$, respectively.

Figure 6 shows the experimental and simulated drag torque results for test case 1 . The model prediction results are in a good agreement with the experimental results. Comparing the results of two test cases suggests that higher flow rate causes higher overall drag torque and vice versa. The reason for this behavior is attributed to the fact that in case of higher flow rate, cavitation is pushed away to higher speeds. Therefore, the critical speed shifts to higher value, and consequently, the drag torque increases. The critical speed refers to the clutch speed where cavitation commences, as a result of which the full ATF film begins to rupture. Thus, it is observed that cavitation commences, accompanied by the film rupture, at lower speed of $500 \mathrm{rpm}$ for low flow rate, whereas it occurs at a higher speed of $700 \mathrm{rpm}$ for high flow rate.

4.2. ATF Temperature. A test is performed for the purpose of evaluating the model behavior with variation in ATF temperature.

The initial value of flow rate of $4 \mathrm{~L} \cdot \mathrm{min}^{-1}$ and ATF temperature of $80^{\circ} \mathrm{C}$ is maintained during the test. The remaining parameters of the test are kept the same as that of the second test (test case 1), as given in Table 7, test case 2, belonging to the model validation group. Thus, using these two tests, the model assessment for two different values 


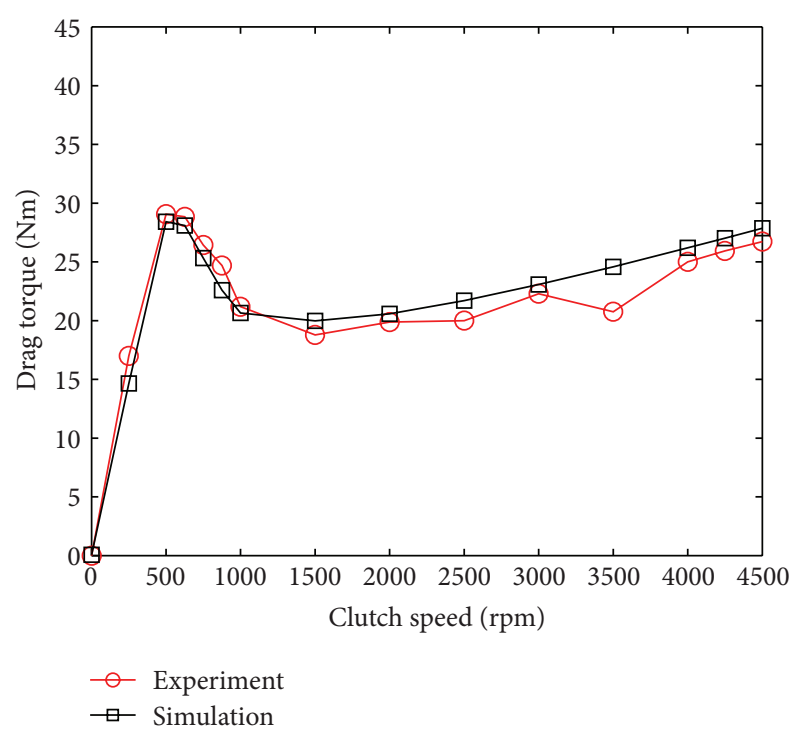

(a)

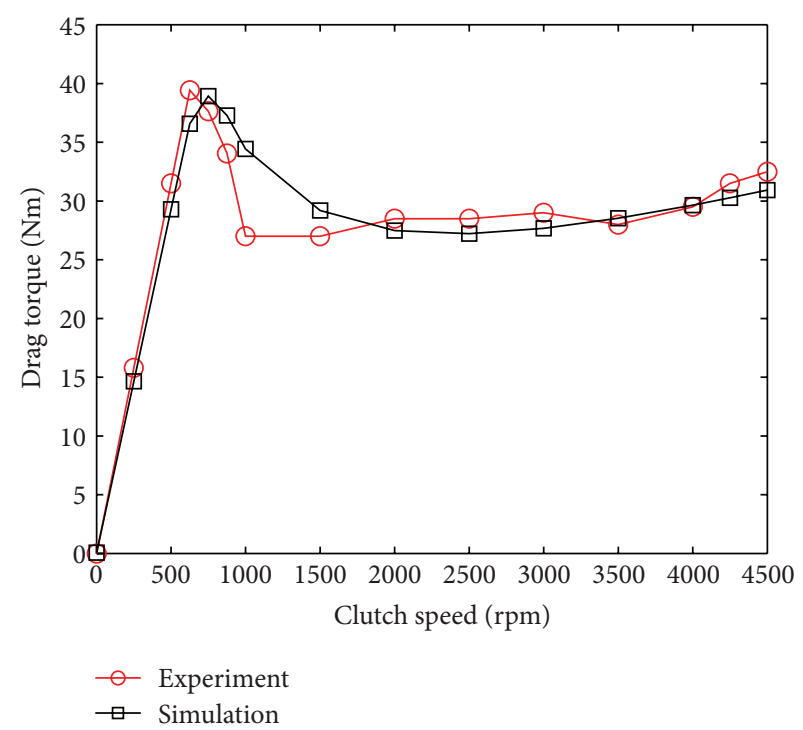

(b)

FIgURE 6: Experimental and simulated drag torque for initial ATF flow rate: (a) $2.2 \mathrm{~L} \cdot \mathrm{min}^{-1}$; (b) $4 \mathrm{~L} \cdot \mathrm{min}^{-1}$.

of ATF temperature $\left(60\right.$ and $\left.80^{\circ} \mathrm{C}\right)$ can be done. The ATF density is $885 \mathrm{Kg} \cdot \mathrm{m}^{-3}$. The ATF and mist dynamic viscosities considered for the simulations are 0.060 and $0.0040 \mathrm{~Pa} \cdot \mathrm{s}$, respectively.

Figure 7 shows the experimental and simulated drag torque results for test case 2 . On comparing the present test result with the second test of test case 1 reveals that with the increase in ATF temperature, the overall drag torque reduces. This reduction is due to the decrease in fluids viscosities with rise in ATF temperature. Another observation is that for both tests, cavitation commences and the rupture of the ATF film gets started at the same critical speed $(700 \mathrm{rpm})$. Following this, the drag torque reduces due to reduction in the amount of ATF film in the gap between the disks. After a drop, the drag torque again increases with further increase in clutch speed. This increase is due to the increasing external flow rate and a slight contribution from the mist film in high-speed region. The results confirm the model capability of predicting drag torque for different magnitudes of ATF temperature.

4.3. Disks' Rotational State. In order to gauge the model potential in estimating the drag torque for different disks' rotational states, two tests are performed. In the first test, the SDs are held stationary while the FDs are rotating (FS0). In contrast, in the second test, both the disks are rotating with the same speed but in opposite directions (OR). The parameters of the tests are given in Table 7, test case 3, belonging to the model validation group. The ATF density is $865 \mathrm{Kg} \cdot \mathrm{m}^{-3}$. The dynamic viscosities of the ATF and mist considered for the simulation are 0.070 and $0.0015 \mathrm{~Pa} \cdot \mathrm{s}$, respectively.

Figure 8 shows the experimental and the simulated drag torque results for FS0 and OR disks' rotational states. The results suggest that the simulation results match closely with the experimental results for both cases. In addition, the

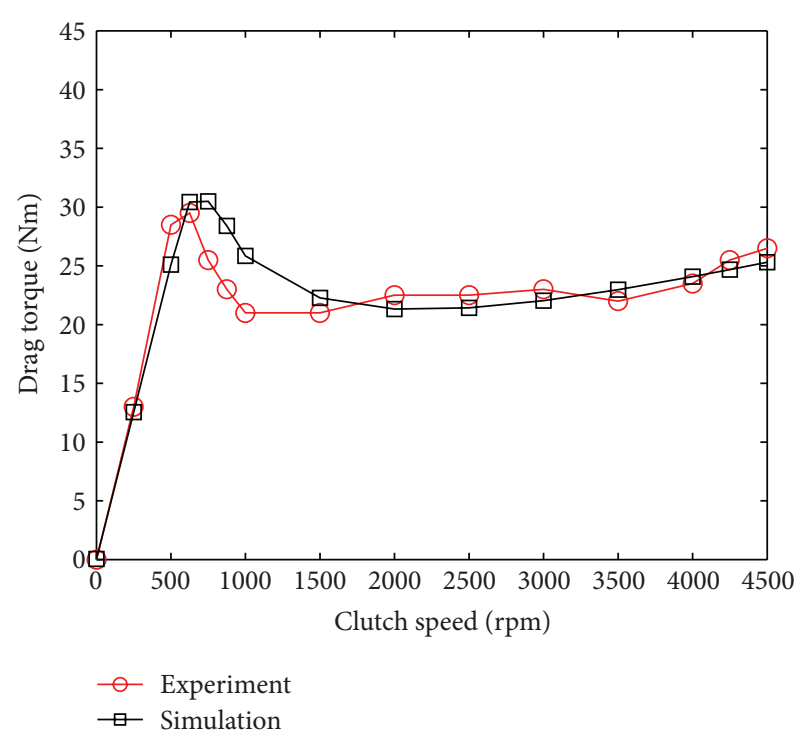

FIGURE 7: Experimental and simulated drag torque for initial ATF flow rate of $4 \mathrm{~L} \cdot \mathrm{min}^{-1}$ and ATF temperature of $80^{\circ} \mathrm{C}$.

disk rotational state OR exhibits higher overall drag torque as compared to disk rotational state FS0. This behavior is due to two reasons. Firstly, in case of disk rotational state $\mathrm{OR}$, the clutch speed is double the case of disk rotational state FS0. As the drag torque is proportional to the clutch speed, the OR rotational case results in a higher overall drag torque as compared to the FS0 rotational case. Secondly, the disk rotational state OR has an inherent tendency to impose lower centrifugal force onto the flow as compared to the disk rotational state FS0 [10]. Consequently, more ATF film is retained in the gap between the disks for OR case as compared to FS0 case. Thus, the OR case generates higher overall drag torque as compared to the FSO case. 


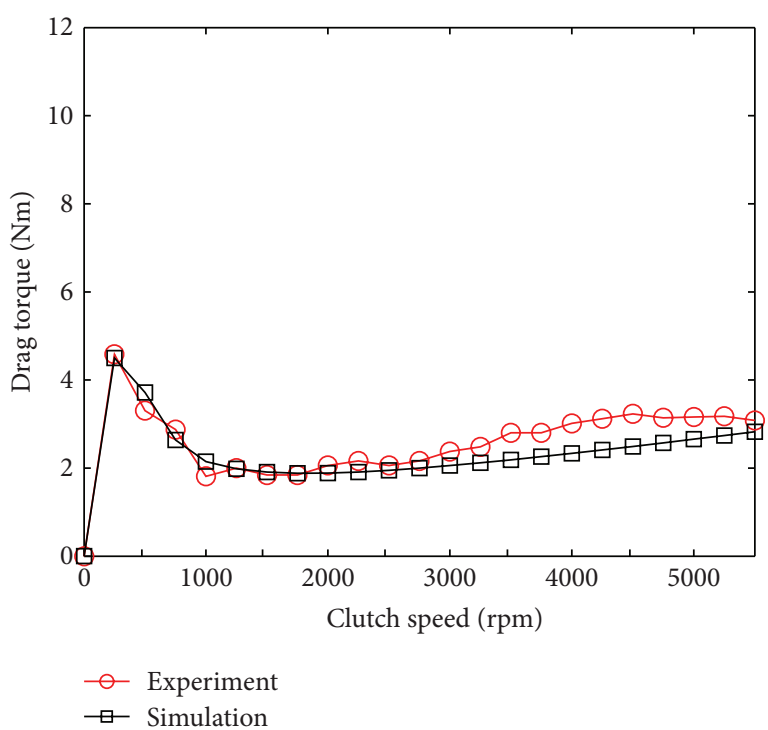

(a)

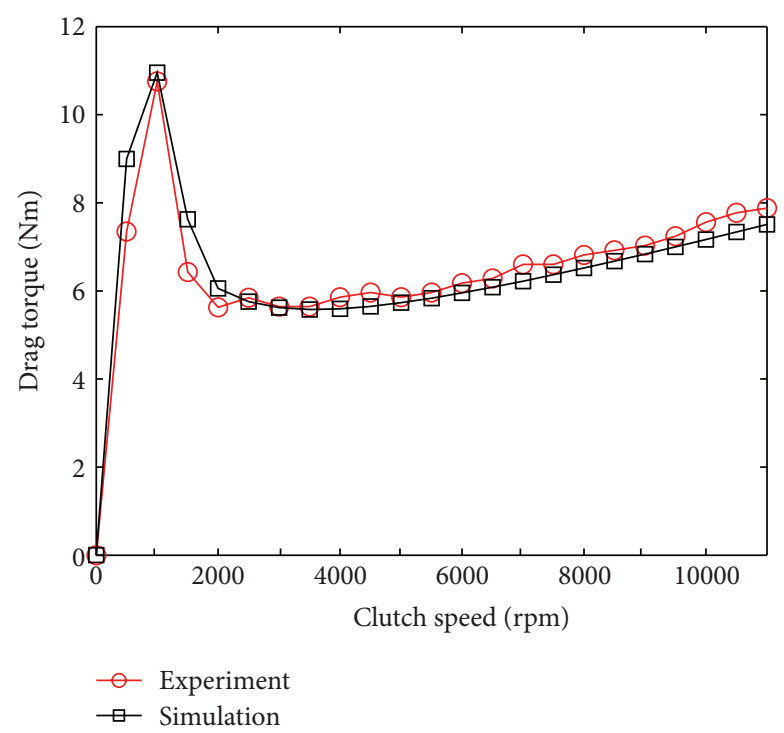

(b)

FIGURE 8: Experimental and simulated drag torque for disks' rotational states: (a) FS0; (b) OR.

Moreover, the critical speed at which drag torque peak occurs or cavitation commences is approximately twice for the OR case as compared to the FSO case.

\section{Experimental Evaluation}

This section discusses the results related to the experimental evaluation of the drag torque performed under real conditions of variable flow rate and disk rotational state for higher clutch speed range. The parameters considered in the present study are ATF flow rate, ATF temperature, disks size, and disks' rotational states (OR, ER, and FS0). Drag torque results are mostly plotted in function of clutch speed (difference in speed of FDs and SDs). However, in some cases for better comparison drag torque is plotted in function of clutch absolute speed, which is equal to the speed of either FDs or SDs.

5.1. Effects of Varying ATF Flow Rate. In order to study the influence of variation in ATF flow rate on drag torque, three tests are conducted. The initial value and rate of increase in ATF flow rate supply in the three tests are maintained to 5.1 (Q-1)-0.0049 (m-1), $6.7(Q-2)-0.0066(m-2)$, and $8.5 \mathrm{~L} \cdot \mathrm{min}^{-1}$ (Q-3)-0.0085 L $\cdot \mathrm{min}^{-1} \mathrm{rpm}^{-1}(m-3)$, respectively. The remaining test specifications are given in Table 7 , test set 1 , belonging to the experimental evaluation group.

Figure 9 shows the experimental results of the test set 1 . The results suggest that for a particular flow rate, at low-speed region there exist a full ATF film in the gap between the disks, and consequently, the drag torque increases with increasing clutch speed up to a certain critical speed. Beyond this critical speed, the drag torque decreases (due to reduction in the ATF film) followed by a slow increase in the high-speed region.

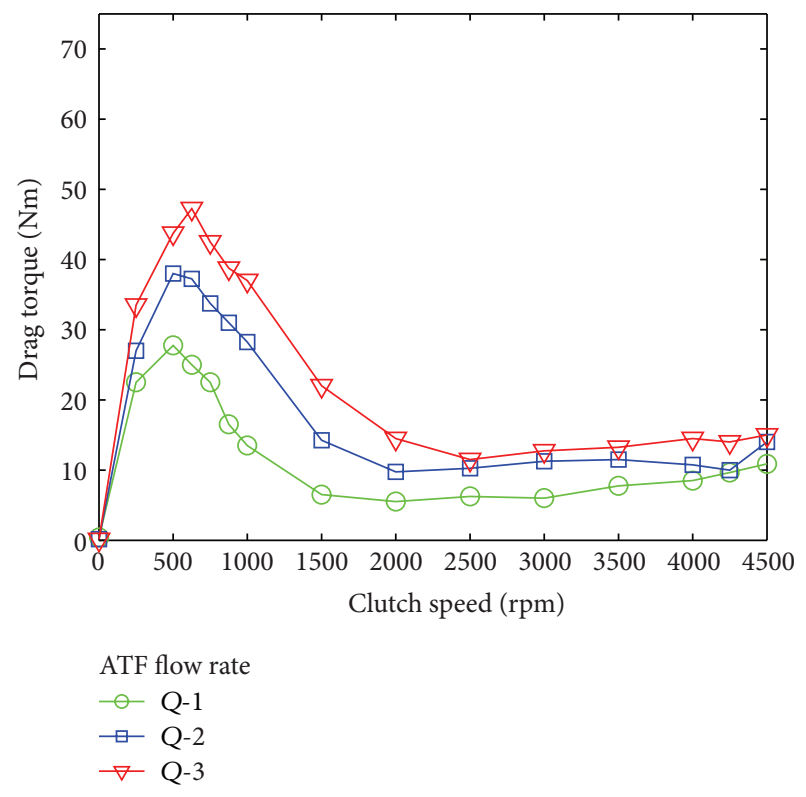

Figure 9: Effects of varying ATF flow rate.

This slow increase in the drag torque is due to the increasing external flow and a slight contribution from the mist film.

The overall drag torque increases throughout the clutch speed range with increasing ATF flow rate. The reason for this behavior is attributed to the fact that with the increase in ATF flow rate, cavitation is pushed away to higher speeds. Therefore, the speed up to which full ATF film is retained increases or the critical speed shifts to higher value, and consequently, the drag torque is higher. The amount by which the drag torque increases is directly proportional to the magnitude of the ATF flow rate as shown in Table 2. 

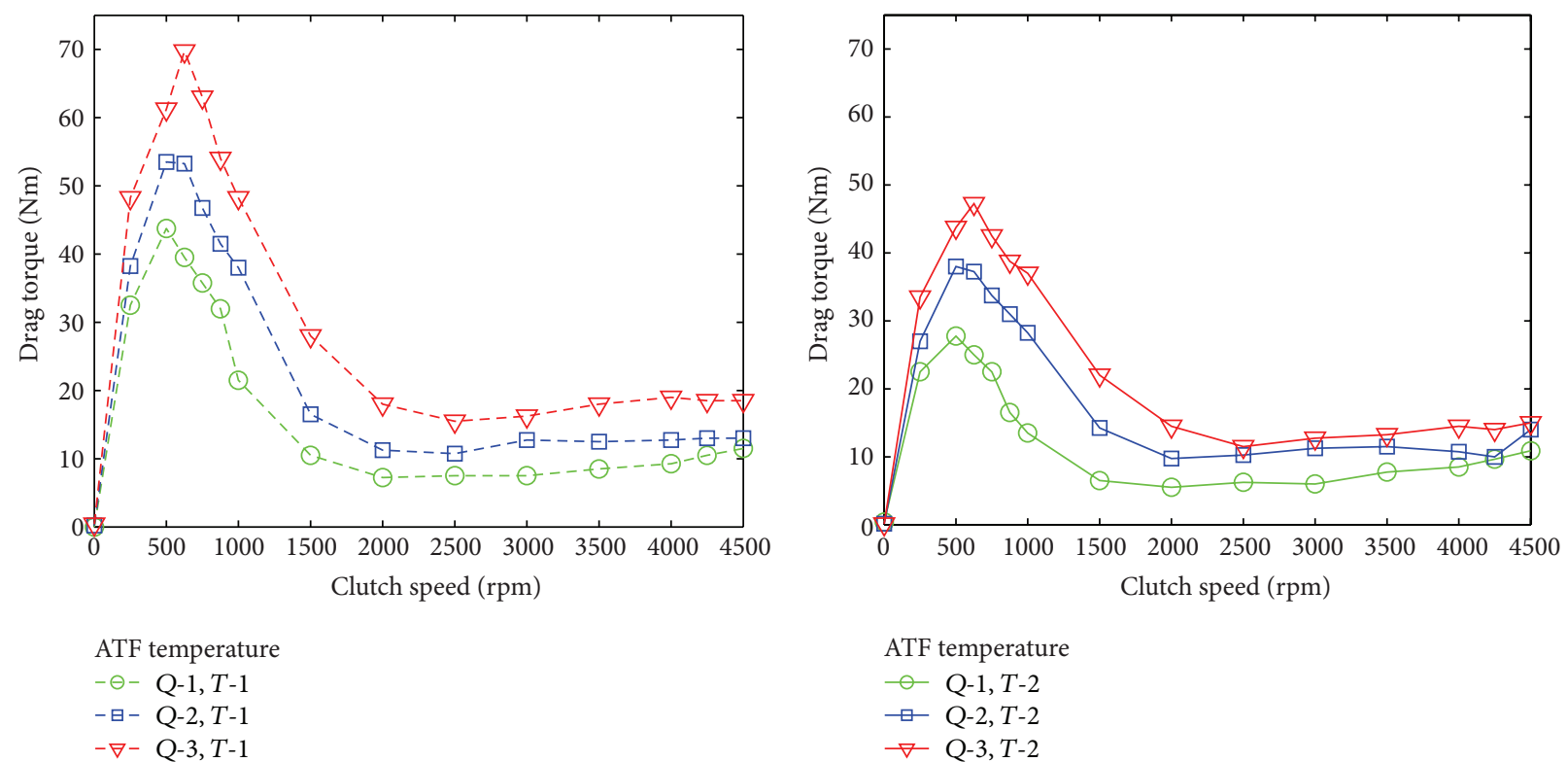

ATF temperature

$-Q-1, T-2$

$\square \mathrm{Q}-2, T-2$

$\rightarrow Q-3, T-2$

(a)

(b)

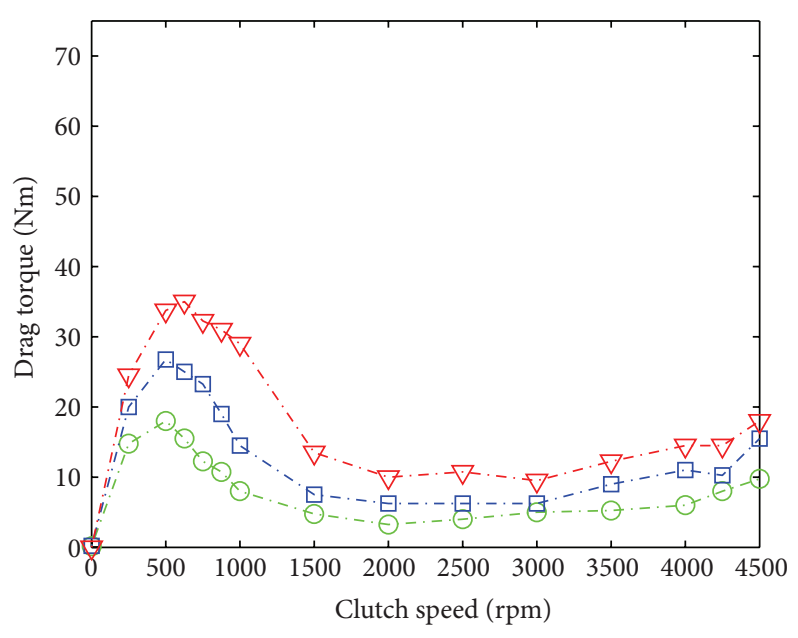

$$
\begin{aligned}
& \text { ATF temperature } \\
& -\bigcirc-\text { Q-1,T-3 } \\
& -\square-\text { Q-2,T-3 } \\
& -\nabla-\text { Q-3,T-3 }
\end{aligned}
$$

(c)

Figure 10: Effects of varying ATF temperature to (a) $40^{\circ} \mathrm{C}$, (b) $60^{\circ} \mathrm{C}$, and (c) $80^{\circ} \mathrm{C}$.

Due to the reason mentioned above, the test results depict that the cavitation commences, accompanied by the film rupture, at a lower critical speed $(500 \mathrm{rpm})$ for the lower flow rate $(Q-1)$, whereas it occurs at a higher critical speed $(625 \mathrm{rpm})$ for higher flow rate $(Q-3)$. The increment in critical speed with the increase in flow rate follows a relation of square root of the relative increase in the flow rate as shown in Table 2.

5.2. Effects of Varying ATF Temperature. In order to study the influence of variation in ATF temperature on the drag torque, three sets of tests are conducted. The ATF temperatures in the three sets of tests are maintained at $40(T-1), 60(T-2)$, and $80^{\circ} \mathrm{C}(T-3)$, respectively. The remaining test specifications are given in Table 7 , test set 2 , belonging to the experimental evaluation group.

Figure 10 presents the experimental results for the test set 2. The results suggest that the drag torque reduces with the rise in ATF temperature up to the start of the slow increasing portion of the drag torque curve (around $2000 \mathrm{rpm}$, in the present plot). This reduction is attributed to the fact that the viscosity of the ATF reduces with the increasing temperature, which in turn lowers the shear forces and the corresponding drag torque. The trend in reduction in the drag torque follows 
TABLE 2: The relationship between the ATF flow rate, drag torque and critical speed.

\begin{tabular}{lccc}
\hline Initial flow rate $\left(\mathrm{L} \cdot \mathrm{min}^{-1}\right)$ & Rate of increase $\left(\mathrm{L} \cdot \mathrm{min}^{-1} \mathrm{rpm}^{-1}\right)$ & Peak drag torque $(\mathrm{Nm})$ & Critical speed $(\mathrm{rpm})$ \\
\hline$Q-1=5.1=X$ & $m-1=0.0049=Y$ & $28 \approx Z$ & $500=S$ \\
$Q-2=6.7=1.3 X$ & $m-2=0.0066=1.3 Y$ & $38 \approx 1.3 Z$ & $550 \approx \sqrt{ }(Q-2 / Q-1) S$ \\
$Q-3=8.5=1.7 X$ & $m-3=0.0085=2.7 Y$ & $48 \approx 1.7 Z$ & $625 \approx \sqrt{ }(Q-3 / Q-1) S$ \\
\hline
\end{tabular}

TABLE 3: The relationship between the ATF temperature, ATF dynamic viscosity, peak drag torque, and critical speed.

\begin{tabular}{|c|c|c|c|}
\hline Temperature $\left({ }^{\circ} \mathrm{C}\right)$ & Dynamic viscosity $(\mathrm{Pa} \cdot \mathrm{s})$ & Peak drag torque $(\mathrm{Nm})$ & Critical speed (rpm) \\
\hline 40 & $0.027=\mu-1=X$ & $70=Y$ & $625=S$ \\
\hline 60 & $0.017=\mu-2=0.7 X$ & $48=0.7 Y$ & $500=0.80 S \approx \sqrt{ }(\mu-2 / \mu-1) S$ \\
\hline 80 & $0.012=\mu-3=0.5 X$ & $35=0.5 Y$ & $450=0.72 S \approx \sqrt{ }(\mu-3 / \mu-1) S$ \\
\hline
\end{tabular}

the trend in reduction in ATF viscosity with the increasing temperature of the ATF. In addition, the experimental results reveal that for a particular flow rate, the critical speed decreases with increasing ATF temperature. This reduction in critical speed follows a relation of the square-root of the relative decrease in the dynamic viscosity of the ATF with increasing ATF temperature as shown in Table 3.

The relationship between the ATF temperature, ATF dynamic viscosity, peak value of the drag torque (considering flow rate Q-3 as an example) and critical speed is given in Table 3.

5.3. Effects of Varying Disks Size. Three tests are conducted for the purpose of evaluating the effect of disk size on the drag torque. The tests specifications are given in Table 7 , test set 3 , belonging to the experimental evaluation group. Figure 11 shows the experimental results of test set 3 . The results suggest that the drag torque increases with increase in the mean radius of the disks. This increase in the drag torque is because of the reason that with the increase in disk mean radius the shearing area and consequently the drag torque increases. This increment in the drag torque follows a relation of fourth power of the relative increase in mean radius of the disks [13] as shown in Table 4.

5.4. Effects of Different Disks' Rotational States. In order to evaluate the effect of different disks' rotational states on the drag torque, three tests are conducted. The test specifications are given in Table 7, test set 4, belonging to the experimental evaluation group. The disks' rotational states considered in the tests are OR, ER, and FS0. Figure 12 presents the experimental results for the test set 4 . The result suggests that the disk rotational state OR exhibit maximum, while the disk rotational state ER exhibit minimum overall drag torque. In addition, for the disk rotational state ER, the critical speed at which the drag torque peak occurs (onset of cavitation) is lower than that of the other two disks' rotational states (OR and FS0). However, all the three disks' rotational states show similar trend in the behavior of the drag torque curve.

The explanation for these effects is as follows. As described previously, the drag torque is proportional to the clutch speed (difference in speed of FDs and SDs) and increases with the amount of the ATF in the gap between
TABLE 4: The relationship between the disk mean radius and drag torque.

\begin{tabular}{lcc}
\hline Clutch & Mean radius $(\mathrm{mm})$ & Peak drag torque $(\mathrm{Nm})$ \\
\hline$C-1$ & $58.5=X$ & $0.17=Y$ \\
$C-2$ & $69=1.18 X$ & $0.30 \approx(1.18)^{4} Y$ \\
$C-3$ & $76.5=1.3 X$ & $0.49=(1.3)^{4} Y$ \\
\hline
\end{tabular}

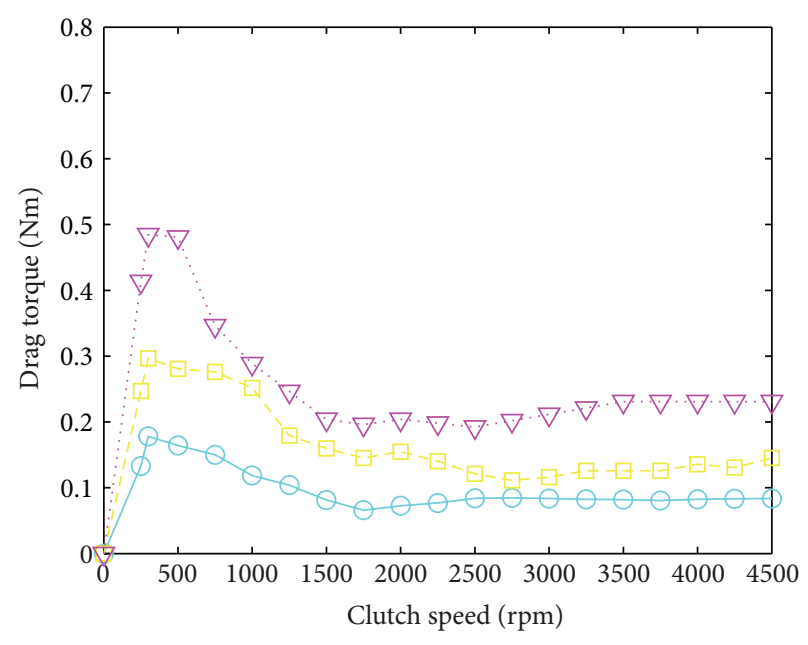

Disks' size
$-\odot$ C-1
$-\boxminus-C-2$
$-\nabla \cdot C-3$

Figure 11: Effects of varying disks' size.

the disks. The amount of ATF film retained in the gap is in turn depends upon the magnitude of centrifugal force acting onto the flow. As reported in [13], the inherent tendency of the different disks' rotational states to impose centrifugal force onto the flow is dependent on the magnitude of term as indicated within bracket in (18). Considering this term and assuming $\omega$ as an absolute speed of rotations of the FDs or the SDs, the relative magnitudes of centrifugal force imposed onto the flow for different disks' rotational states are given in Table 5 .

From Table 5, it is evident that theoretically OR case exhibits maximum clutch speed whereas ER case results in 
TABLE 5: The relationship between the critical clutch speed and the relative magnitude of centrifugal force imposed onto the flow for different disks' rotational states.

\begin{tabular}{lccc}
\hline $\begin{array}{l}\text { Disks' } \\
\text { rotational } \\
\text { state }\end{array}$ & $\begin{array}{c}\text { Clutch } \\
\text { speed }\end{array}$ & $\begin{array}{c}\text { Relative } \\
\text { centrifugal } \\
\text { force }\end{array}$ & $\begin{array}{c}\text { Critical speed at which } \\
\text { drag torque peak occurs }\end{array}$ \\
\hline OR & $2 \omega$ & $0.2 \omega^{2}$ & OR $=2.2 \omega \approx 2 \mathrm{ER}$ \\
ER & 0 & $\omega^{2}$ & ER $=\omega$ \\
FS0 & $\omega$ & $0.3 \omega^{2}$ & FS0 $=1.82 \omega \approx 2 \mathrm{ER}$ \\
\hline
\end{tabular}

TABLE 6: Clutch geometrical details.

\begin{tabular}{lcccc}
\hline Clutch ID & $\begin{array}{c}\text { Inner radius } \\
(\mathrm{mm})\end{array}$ & $\begin{array}{c}\text { Outer radius } \\
(\mathrm{mm})\end{array}$ & $\begin{array}{c}\text { Gap height } \\
(\mathrm{mm})\end{array}$ & $\begin{array}{c}\text { Area ratio } \\
(-)\end{array}$ \\
\hline$C-1$ & 50 & 67 & 0.32 & 0.80 \\
$C-2$ & 57 & 81 & 0.32 & 0.80 \\
$C-3$ & 57 & 96 & 0.32 & 0.85 \\
$C-4$ & 55 & 81 & 0.38 & 0.80 \\
\hline
\end{tabular}

zero clutch speed (however during measurements, speed is never perfectly controlled and consequently results in some minor amount of clutch speed, even for the ER case). In addition, the inherent tendency to impose the centrifugal force onto the flow is highest for the ER case and lowest for the OR case. Thus, the amount of ATF retained in the gap between the disks is greatest for the OR case and least for the ER case as shown schematically in Figure 13. Thus, taking into account, the clutch speed and the amount of ATF retained in the gap, the disk rotational state OR results in highest, whereas the disk rotational state ER results in least overall drag torque. Furthermore, under same test conditions, the drag torque peak or the rupture of the full ATF film (due to onset of cavitation) should happen approximately at twice the absolute clutch speed for OR and FS0 cases as compared to ER case (see fourth column). This appears to be approximately the case in Figure 12.

\section{Conclusions}

A mathematical model is presented in this paper to estimate the drag torque in disengaged multidisks' wet clutches. The model calculates the flow velocity and pressure fields and describes the flow development. Increased centrifugal force at higher speed causes a positive pressure gradient zone (theoretically) starting from the outer radius in inward direction, which leads to cavitation. Due to cavitation, the ATF film is ruptured from the outer radius in inward direction with the formation of rivulets and a mist film in between the rivulets. The model quantifies the volume fraction of the fluids as a function of clutch speed. The drag torque estimated by the model is the sum of drag torque due to shearing of the ATF (both in the continuous and the ruptured sections) and in the mist film. The model validation results suggest that the model is capable of predicting the drag torque under real clutch conditions of variable flow rate and disks' rotational states for higher clutch speed range. The simplicity of the model makes it suitable for online optimization and control.

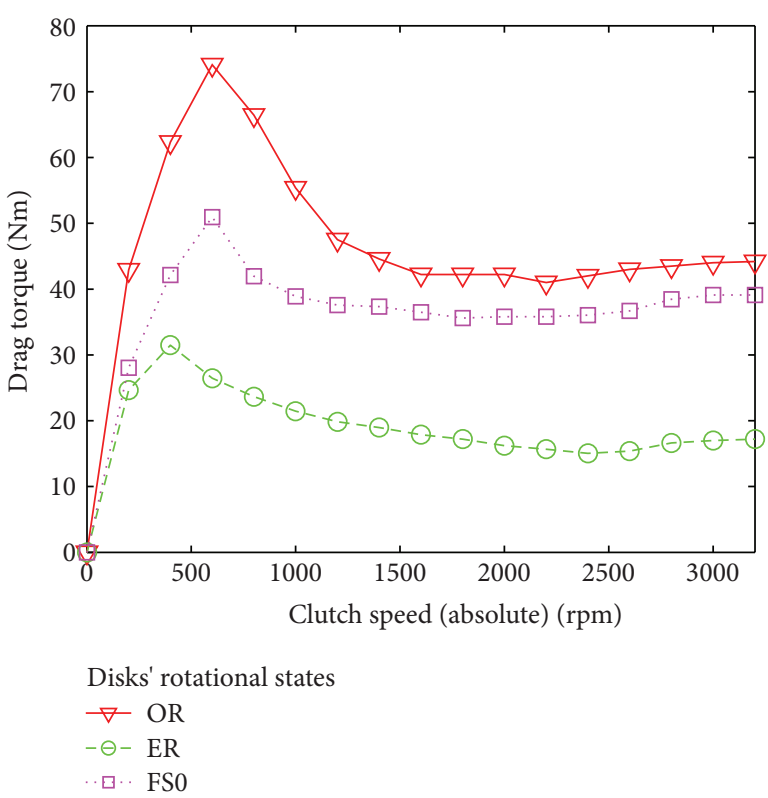

FIGURE 12: Effects of different disks' rotational states.

On the basis of experimental results, the qualitative relationship between the drag torque and clutch speed is constructed as shown in Figure 14. In order to describe the relationship clearly, the curve has been divided into three speed zones, namely low-, mid-, and high-speed zones. The behavior of the drag torque in each zone is discussed below [13].

Low-Speed Zone. In the low-speed region, the centrifugalforce induced flow is everywhere less than the feed flow. This results in a positive pressure distribution throughout the ATF film. In such situations, there exists a full continuous ATF film in the gap between the disks, and the flow is single phase. Drag torque increases with increasing clutch speed in this zone.

Mid-Speed Zone. With the increase in speed (in mid-speed region), the centrifugal-force-induced flow increases and becomes, near the outer radius, greater than the feed flow. In order to satisfy the flow continuity, the pressure gradient should become positive in that part of the film. A negative pressure zone starting from the outer radius in inward direction is thus (theoretically) formed. Due to the negative pressure and also to satisfy the law of conservation of mass, cavitation commences, and the ATF film is ruptured from the outer radius in inward direction with the formation of rivulets and a mist film in between the rivulets. In this zone, the drag torque reduces due to the reduction in the amount of ATF flow, and also because the effective viscosity of mist is low as compared to the ATF.

High-Speed Zone. In the high-speed region, there is a further increase in the centrifugal force, so the centrifugal-forceinduced flow is everywhere greater or equal to the feed flow. This leads to a theoretically positive pressure gradient (negative pressure) throughout the fluids in the gap. In such 
TABLE 7: Summary of the test specifications for model validation and experimental evaluation.

\begin{tabular}{|c|c|c|c|c|c|c|c|c|}
\hline $\begin{array}{l}\text { Test } \\
\text { case/set }\end{array}$ & $\begin{array}{l}\text { Parameters } \\
\text { varied }\end{array}$ & $\begin{array}{l}\text { Clutch } \\
\text { ID }(-)\end{array}$ & $\begin{array}{c}\text { No. of } \\
\text { interfaces } \\
(-)\end{array}$ & $\begin{array}{l}\text { Initial flow } \\
\text { rate }\left(\mathrm{L} \cdot \mathrm{min}^{-1}\right)\end{array}$ & $\begin{array}{c}\text { Rate of increase in } \\
\text { flow rate } \\
\left(\mathrm{L} \cdot \mathrm{min}^{-1} \mathrm{rpm}^{-1}\right)\end{array}$ & $\begin{array}{l}\text { ATF tem- } \\
\text { perature } \\
\left({ }^{\circ} \mathrm{C}\right)\end{array}$ & $\begin{array}{l}\text { FDs speed } \\
\quad(\mathrm{rpm})\end{array}$ & SDs speed (rpm) \\
\hline \multicolumn{9}{|c|}{ Model validation } \\
\hline 1 & ATF flow rate & $C-3$ & 26 & $\begin{array}{l}Q-1=2.2 \\
Q-2=4\end{array}$ & $\begin{array}{l}m-1=0.0012 \\
m-2=0.0012\end{array}$ & 60 & $0-4500$ & 0 \\
\hline 2 & $\begin{array}{c}\text { ATF } \\
\text { temperature }\end{array}$ & $C-3$ & 26 & 4 & 0.0012 & 80 & $0-4500$ & 0 \\
\hline 3 & $\begin{array}{l}\text { Disks' rotational } \\
\text { state }\end{array}$ & $C-4$ & 22 & 0.75 & 0.0002 & 60 & $\begin{aligned} \mathrm{FS} 0 & =0-5500 \\
\mathrm{OR} & =0-5500\end{aligned}$ & $\begin{array}{c}\text { FS0 }=0 \\
\text { OR }=0-(-5500)\end{array}$ \\
\hline \multicolumn{9}{|c|}{ Experimental evaluation } \\
\hline 1 & ATF flow rate & $C-3$ & 26 & $\begin{array}{l}Q-1=5.1 \\
Q-2=6.7 \\
Q-3=8.5\end{array}$ & $\begin{array}{c}m-1=0.0049 \\
m-2=0.006 \\
m-3=0.0085\end{array}$ & 60 & $0-4500$ & 0 \\
\hline 2 & $\begin{array}{c}\text { ATF } \\
\text { temperature }\end{array}$ & $C-3$ & 26 & $5.1-6.7-8.5$ & $\begin{array}{c}0.0049-0.0066- \\
0.0085\end{array}$ & $\begin{array}{l}T-1=40 \\
T-2=60 \\
T-3=80\end{array}$ & $0-4500$ & 0 \\
\hline 3 & Disks' size & $\begin{array}{l}C-1 \\
C-2 \\
C-3\end{array}$ & 1 & 1.7 & 0.0016 & 60 & $0-4500$ & 0 \\
\hline 4 & $\begin{array}{c}\text { Disks' rotational } \\
\text { state }\end{array}$ & $C-3$ & 26 & 8.5 & 0.0085 & 80 & $\begin{array}{l}\mathrm{ER}=0-3200 \\
\mathrm{OR}=0-3200 \\
\mathrm{FS} 0=0-3200\end{array}$ & $\begin{array}{c}\mathrm{ER}=0-3200 \\
\mathrm{OR}=0-(-3200) \\
\mathrm{FS} 0=0\end{array}$ \\
\hline
\end{tabular}
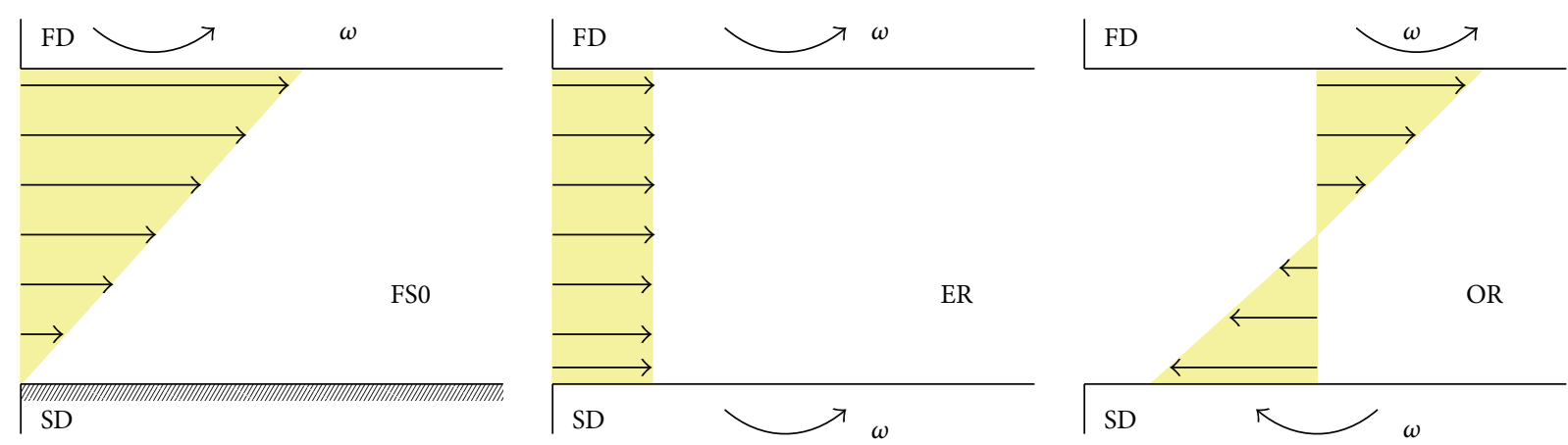

FIGURE 13: Schematic of the qualitative flow configuration after cavitation for disks' rotational states FS0, ER, and OR [13].

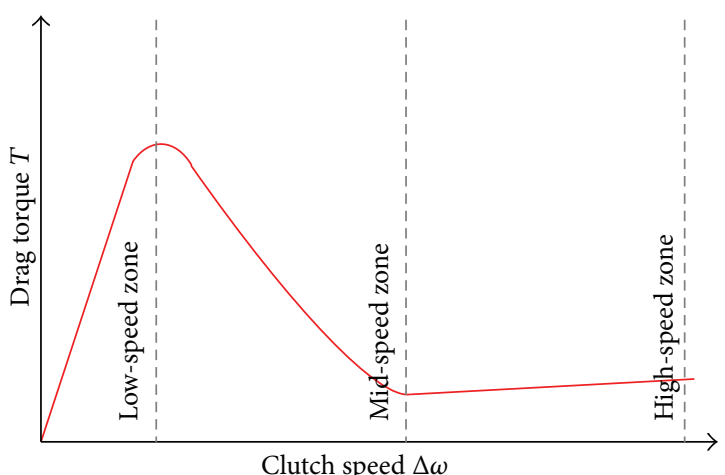

FIGURE 14: The qualitative relationship between the drag torque and clutch speed [13].

a situation, there exists a discontinuous ATF film surrounded by the mist film, which gets thinner with increasing clutch speed. In this speed zone, there is a slow increase in the drag torque, partially contributed due to the ATF and the mist film.

Some conclusions drawn from the experimental evaluation of the drag torque in disengaged wet clutches are listed below.

(i) Increasing ATF flow rate boosts the overall drag torque. This is due to the reason that for higher flow rate cavitation is pushed away to higher speed. Therefore, the speed up to which full ATF film is retained increases resulting in higher drag torque. This buildup of the drag torque is directly proportional to the increase in the flow rate, whereas the increment in the critical speed with the increase in flow rate follows a relation of square root of the relative increase in the flow rate.

(ii) With the increase in ATF temperature, the overall drag torque reduces due to the reduction in ATF 
dynamic viscosity. In addition, it is observed that the variation in the ATF temperature has no significant influence on the critical speed. Further, the trend in reduction in the drag torque follows the same trend as that of reduction in the ATF viscosity with rise in ATF temperature.

(iii) The drag torque increases with the increase in disk means radius due to the gain in shearing area. In addition, the increment in the drag torque follows a relation of fourth power of the relative increase in the mean radius.

(iv) Under same test conditions, the disk rotational state OR exhibits greatest, while the disk rotational state ER exhibits least overall drag torque. In addition, in OR and FS0 cases, the drag torque peak occurs approximately at twice the absolute clutch speed as compared to ER case. This is due to the fact that the OR disk rotational state exhibits highest value of clutch speed and has inherent tendency to impose least centrifugal force onto the flow and thus retains full ATF film up to higher clutch speed.

\section{Nomenclature}

$\alpha: \quad$ A sector angle of the complete film including mist film

$\beta$ : $\quad$ Mist film angle

$\gamma: \quad$ Area ratio

$\phi: \quad$ Fraction of the ATF film

$\rho: \quad$ ATF density

$\mu: \quad$ Dynamic viscosity

$\mu_{\mathrm{ATF}}:$ ATF dynamic viscosity

$\mu_{\text {mist }}$ : Mist dynamic viscosity

$\omega_{1}, \omega_{2}$ : Angular speed of disks

$\Delta \omega: \quad$ Clutch speed

$\tau_{z \theta}: \quad$ Shear stress acting on the disk

$h$ : $\quad$ Gap height

$m$ : $\quad$ Rate of increase in flow rate

$N$ : Number of frictional interface

$P_{a}: \quad$ Ambient pressure

$P: \quad$ Flow pressure

$P_{\text {cav }}: \quad$ Cavitation pressure

$Q_{a}: \quad$ Actual flow rate

$Q_{c}: \quad$ Centrifugal force-induced flow

$Q_{p}: \quad$ Poiseuille force-induced flow

$Q_{i}$ : Initial flow rate

$r, \theta, z$ : Radial, circumferential, and axial coordinates

$r_{i}, r_{o}:$ Inner and outer radii of disk

$r^{*}: \quad$ Critical radius

$T_{f a}: \quad$ Drag torque due to ATF film in continuous section

$T_{r a}: \quad$ Drag torque due to ATF film in ruptured section

$T_{r m}$ : Drag torque due to mist film in ruptured section
$T: \quad$ Total drag torque

$V_{r}, V_{\theta}, V_{z}$ : Flow velocity in radial, circumferential, and axial directions.

\section{Abbreviations}

AT: Automatic transmission

ATF: Automatic transmission fluid

CFD: Computational fluid dynamics

FD: Friction disk

SD: Separator disk

ER: Equal rotation (both FDs and SDs rotate at same speed and in same direction)

FS0: SDs are held stationary while FDs rotate

OR: Opposite rotation (both FDs and SDs rotate at same speed but in opposite direction)

VOF: Volume of fluid.

\section{Acknowledgments}

The authors wish to thank Dr. M. Versteyhe, E. Taskiran, and K. Callier of Dana Spicer Off-Highway Products Division, Bruges, Belgium, for their support in the measurement campaign. Financial support by the Belgian Programme on Interuniversity Attraction Poles, initiated by the Belgian Federal Science Policy Office (DYSCO), and KU Leuven Industrial Research Grant are gratefully acknowledged. The first author would like to thank the European Commission EMECW Lot 15, through which he receives scholarship grant for his research.

\section{References}

[1] H. Kitabayashi, C. Li, and H. Hiraki, "Analysis of various factors affecting drag torque in multiple-pate wet clutches," in Proceedings of the JSAE International Spring Fuels and Lubricant Meeting, pp. 1-6, Yokohama, Japan, May 2003.

[2] Y. Kato, T. Murasugi, H. Hirano, and T. Shibayama, "Fuel economy improvement through tribological analysis of the wet clutches and brakes of an automatic transmission," Society of Automotive Engineers of Japan, vol. 16, no. 12, pp. 57-60, 1993.

[3] Y. Yuan, E. A. Liu, J. Hill, and Q. Zou, "An improved hydrodynamic model for open wet transmission clutches," Journal of Fluids Engineering, Transactions of the ASME, vol. 129, no. 3, pp. 333-337, 2007.

[4] C. R. Aphale, J. Cho, W. W. Schultz, S. L. Ceccio, T. Yoshioka, and H. Hiraki, "Modeling and parametric study of torque in open clutch plates," Journal of Tribology, vol. 128, no. 2, pp. 422-430, 2006.

[5] S. Yuan, Z. Peng, and C. Jing, "Experimental research and mathematical model of drag torque in single-plate wet clutch," Chinese Journal of Mechanical Engineering, vol. 24, no. 1, pp. 9197, 2011.

[6] S. Yuan, K. Guo, J. Hu, and Z. Peng, "Study on aeration for disengaged wet clutches using a two-phase flow model," ASME Journal of Fluids Engineering, vol. 132, no. 11, Article ID 111304, 2010.

[7] C. W. Schade, "Effects of transmission fluid on clutch performance," Society of Automotive Engineer, no. 710734, 1971. 
[8] R. L. Fish, "Using the SAE \#2 machine to evaluate wet clutch drag losses," Society of Automotive Engineer, no. 910803, 1991.

[9] F. A. Lloyd, "Parameters contributing to power loss in disengaged wet clutches," Society of Automotive Engineer, no. 740676, 1974.

[10] S. Iqbal, F. Al-Bender, B. Pluymers, and W. Desmet, "Model for predicting drag torque in open wet clutches," ASME Journal of Fluid Engineering. In press.

[11] O. Reynolds, "On the theory of lubrication and its application to Beauchamp Tower's experiments, including an experimental determination of the viscosity of olive oil," Philosophical Transactions of the Royal Society of London A, vol. 177, pp. 157-233.

[12] S. Iqbal, T. Janssens, W. Desmet, and F. Al-Bender, "Transmitted power and energy flow behavior of degrading wet friction clutches," International Journal of Applied Research in Mechanical Engineering, vol. 2, no. 1, pp. 94-100, 2012.

[13] S. Iqbal, F. Al-Bender, B. Pluymers, and W. Desmet, "Experimental characterization of drag torque in open multi-disks wet clutches," SAE Fuels and Lubricant Journal. Under review. 

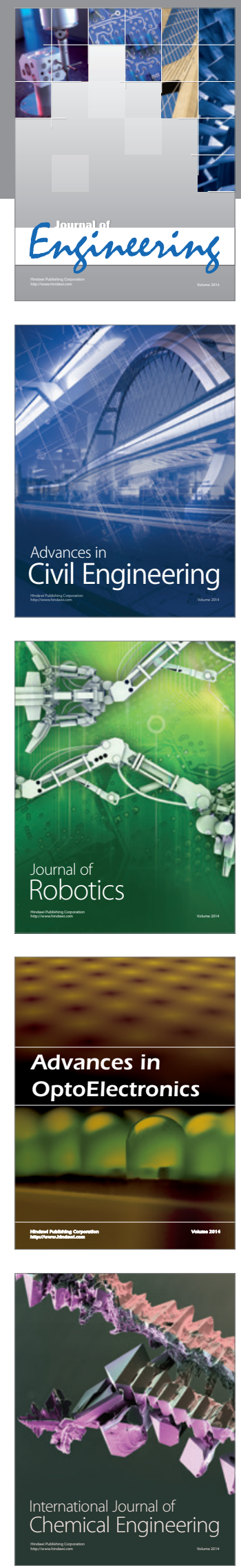

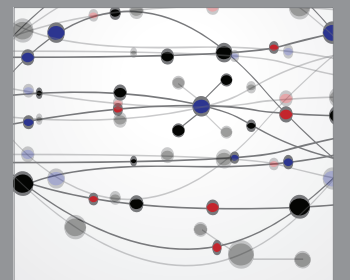

The Scientific World Journal
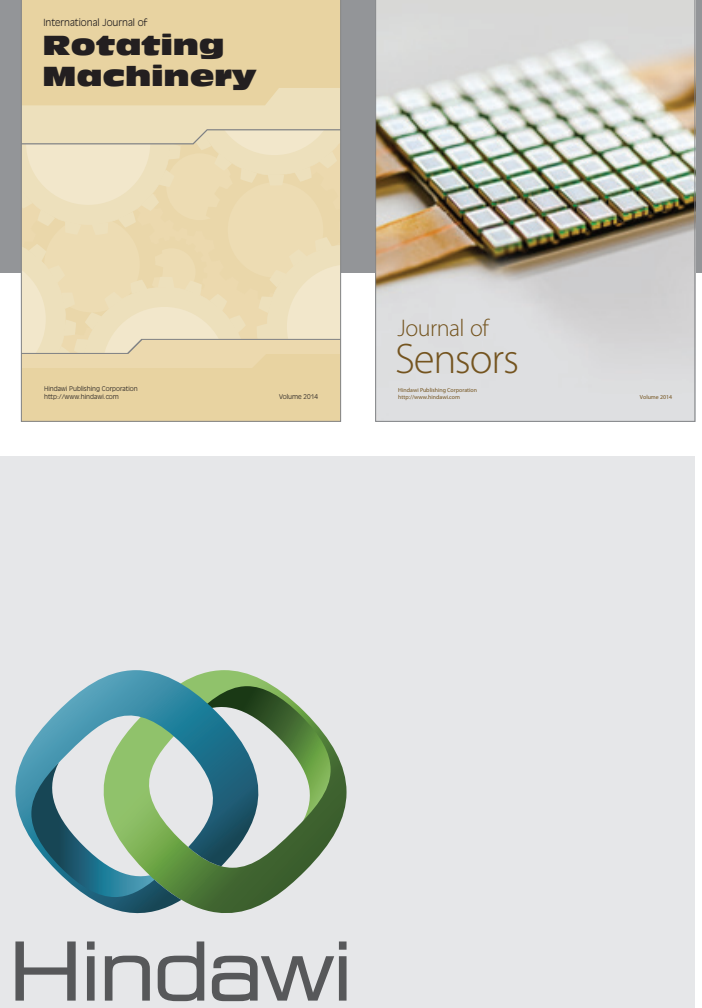

Submit your manuscripts at http://www.hindawi.com
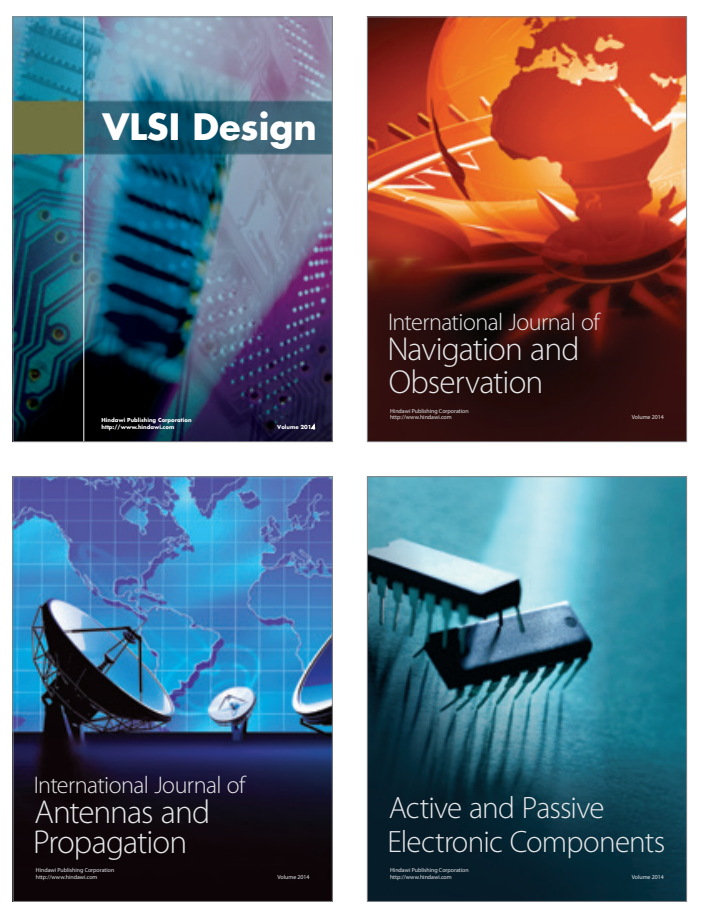
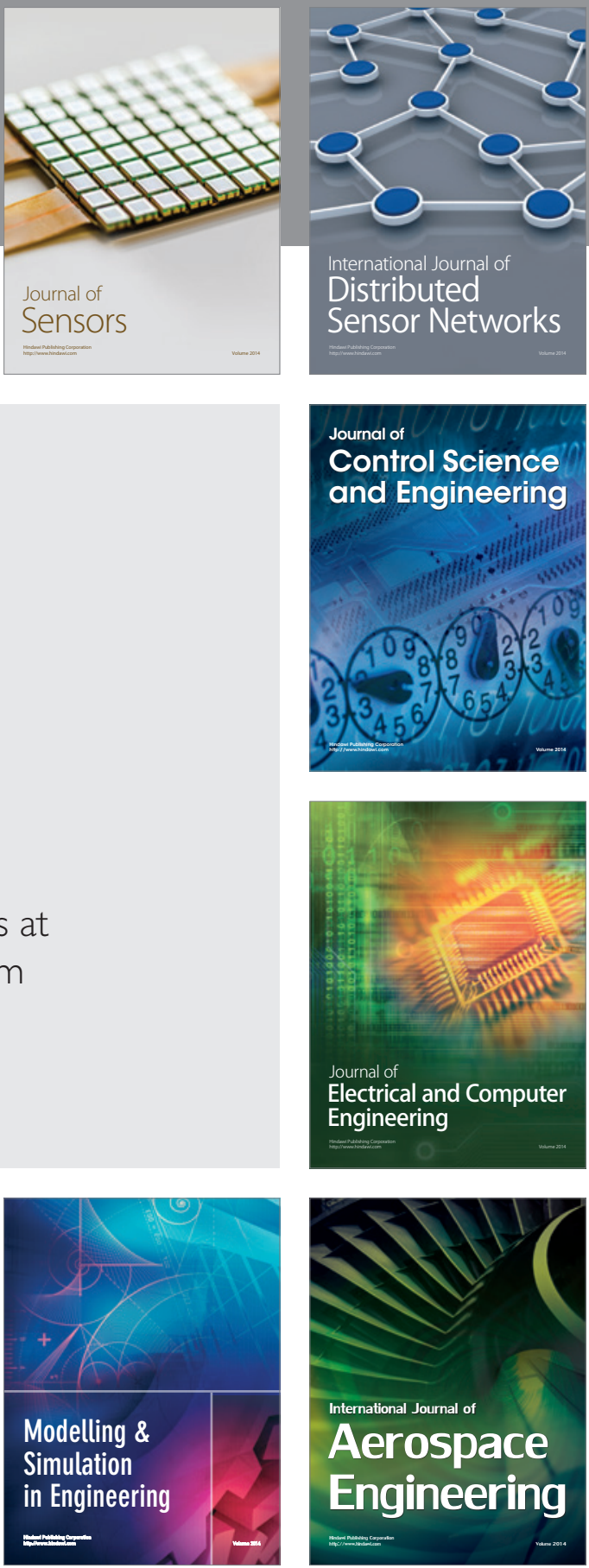

Journal of

Control Science

and Engineering
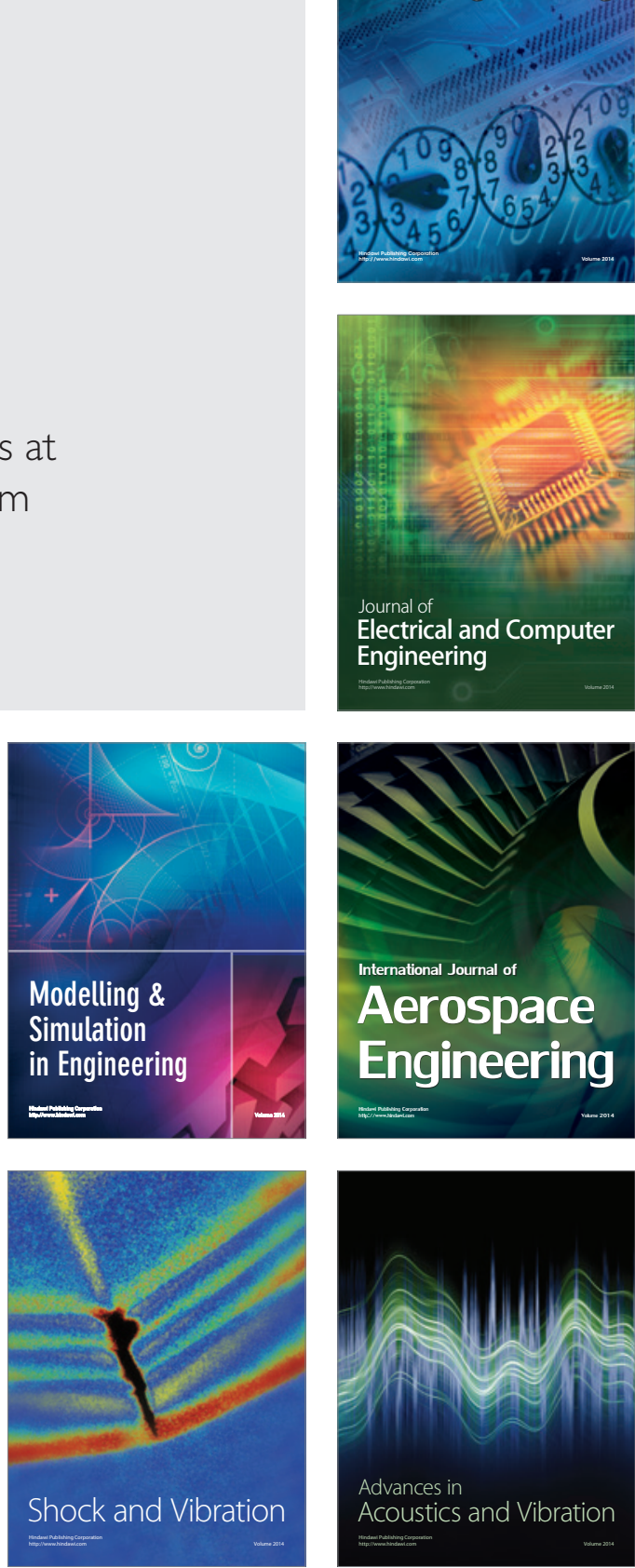